Int. Journal of Game Theory, Vol. 8, Issue 3, page 133-164. (Physica-Verlag, Vienna.

\title{
On Coalition Formation: A Game-Theoretical Approach ${ }^{1}$ )
}

\author{
By P.P. Shenoy, Lawrence ${ }^{2}$ )
}

\begin{abstract}
This paper deals with the question of coalition formation in $n$-person cooperative games. Two abstract game models of coalition formation are proposed. We then study the core and the dynamic solution of these abstract games. These models assume that there is a rule governing the allocation of payoffs to each player in each coalition structure called a payoff solution concept. The predictions of these models are characterized for the special case of games with side payments using various payoff solution concepts such as the individually rational payoffs, the core, the Shapley value and the bargaining set $M^{(i)}$. Some modifications of these models are also discussed.
\end{abstract}

\section{Introduction}

The theory of $n$-person cooperative games is a mathematical theory of coalition behavior. A fundamental problem posed in game theory is to determine what outcomes are likely to occur if a game is played by "rational players". I.e. given an $n$-person cooperative game, it is natural to inquire (1) what will be the final allocation of payoffs to each of the players and (2) which of the possible coalitions can be expected to form. These two aspects of coalition behavior are closely related. The final allocation of pay. offs to each of the players depend on the coalitions that finally form, and the coalitions that finally form depend on the available payoffs to each player in each of these coalitions. Since the publication in 1944 of the monumental work Theory of Games and Economic Behavior by von Neumann/Morgenstern [1944], most of the research in $n$-person game theory has been concerned explicitly with predicting players' payoff and only implicitly (if at all) with predicting which coalitions shall form. In this paper, the primary emphasis is on the second aspect of coalition behavior, namely the formation of coalitions. Two models of coalition formation are proposed based on the theory of $n$-person games. As in most of game theory, our models are normative and use only endogenous arguments, that is, only information contained in the characteristic function is used.

\footnotetext{
1) This research was supported in part by the Office of Naval Research under Contract N00014-75-C-0678 and the National Science Foundation under Grants MPS75-02024 and MCS77-03984 at Cornell University and also by the United States Army under Contract No. DAAG-29-75-C-0024 and the National Science Foundation under Grant No. MCS75-17385 A01 at the University of Wisconsin at Madison. The author is grateful to Professor William F. Lucas under whose guidance the research was conducted and to Professor Louis J. Billera for many helpful discussions.

2) Prof. P.P. Shenoy, The School of Business, The University of Kansas, Lawrence, Kansas 66045 , USA.
} 
A brief review of abstract games and its solutions is presented in section 2. In Section 3 , two abstract game models of coalition formation are proposed. In one ap. proach, payoff allocations and coalition structures are modeled as the outcomes of an abstract game on which an appropriate domination relation is defined. In another approach, coalition structures alone are modeled as outcomes. In both cases, we study the core and the dynamic solution of the abstract game. The two models are then ccmpared. Section 4 deals with the representation of the models by means of digraphs. In Section 5-8, the solutions of the abstract games are characterized for the special case of games with side payments using various payoff solution concepts such as the individually rational payoffs, the core, the Shapley value and the bargaining set $M_{1}^{(i)}$. Finally in Section 9, we discuss possible modifications in the definition of the domination relation in the case where coalition structures alone are modeled as outcomes.

\section{Abstract Games and their Solutions}

An abstract game is a pair ( $X$, dom) where $X$ is an arbitrary set whose members are called outcomes of the game, and dom is an arbitrary binary relation defined on $X$ and is called domination. An outcome $x \in X$ is said to be accessible from an outcome $y \in X$, denoted by $x \leftarrow y$ (or $y \rightarrow x$ ), if there exists outcomes $z_{0}=x, z_{1}, \ldots, z_{m-1}$, $z_{m}=y$, where $m$ is a positive integer such that

$$
x=z_{0} \operatorname{dom} z_{1} \operatorname{dom} z_{2} \operatorname{dom} \ldots \operatorname{dom} z_{m-1} \operatorname{dom} z_{m}=y .
$$

Also assume $x \leftarrow x$, i.e. an outcome is accessible from itself. Clearly the binary relation accessible is reflexive and transitive. Two outcomes $x$ and $y$ which are accessible to each other are said to communicate and we write this as $x \leftrightarrow y$. Since the relation accessible is transitive and reflexive, it follows that

Proposition 2.1. Communication is an equivalence relation.

We can now partition the set $X$ into equivalence classes. Two outcomes are in the same equivalence class if they communicate with each other.

The set

$$
\operatorname{Dom}(x)=\{y \in X: x \operatorname{dom} y\}
$$

is called the dominion of $x$. Also, we define the dominion of any subset $A \subset X$ by

$$
\operatorname{Dom}(A)=\bigcup_{x \in A} \operatorname{Dom}(x)
$$

The core C [due to Gillies; Shapley, 1953] of an abstract game is defined to be the set of undominated outcomes. I.e.

$$
C=X-\operatorname{Dom}(X) \text {. }
$$

We can rewrite the definition of the core in terms of the relation accessible as follows. 


$$
C=\{x \in X: \forall y \in X, y \neq x, \text { we have } y+x\} .
$$

I.e., in the terminology of Markov chains, the core is the set of all absorbing outcomes. Note that each outcomes in the core (if nonempty) is an equivalence class by itself.

An elementary dynamic solution (elem. $d$-solution) of an abstract game is a set $S \subset X$ such that

and

$$
\text { if } x \in S, y \in X-S \text { then } y+x,
$$

if $x, y \in S, \quad$ then $x \leftarrow y$ and $y \leftarrow x$.

It can easily be shown that

Proposition 2.2. An elem. $d$-solution is an equivalence class.

The converse, however, is not always true. Condition (2.6) requires $S$ to be (in the terminology of Markov chains) a non-transient (recurrent, persistent) equivalence class.

The dynamic solution ( $d$-solution) $P$ of an abstract game is the union of all distinct elem. $d$-solutions. I.e.

$$
P=\cup\{S \subset X: S \text { is an elem. } d \text {-solution }\} .
$$

We have the following easy result.

Proposition 2.3. For all abstract games, $C \subset P$.

It is clear that the dynamic solution always exists and is unique. However, it may sometimes be empty. However, if $X$ is a finite set, we have the following two results.

Lemma 2.4. If $X$ is a finite set, then $P$ is the $d$-solution if and only if $P$ satisfies

$$
\begin{aligned}
& \forall x, y \in P, y \leftarrow x \text { iff } x \leftarrow y . \\
& \text { If } x \in P, y \in X-P, \text { then } y+x .
\end{aligned}
$$

And

$$
\text { If } y \in X-P \text {, then } \exists x \in P \text { such that } x \leftarrow y \text {. }
$$

Theorem 2.5. If $X$ is a finite set, then the $d$-solution is always nonempty.

For proofs of these assertions and for a behavioral interpretation of the dynamic solution, see Shenoy [1977a]. The dynamic solution has also been defined independ. ently by Kalai/Pazner/Schmeidler [1976].

\section{The Models}

We shall first introduce some notation and definitions. Let $N=\{1, \ldots, n\}$ denote the set of players. Let $\Gamma$ be an $n$-person cooperative game (with side payments, without side payments or a game in partition function form). Let $2^{N}$ denote the set of all sub- 
sets (coalitions) of $N$ and $\Pi$ denote the set of all partitions (coalition structures, c.s.) of $N$. Let $S: \Pi \rightarrow 2^{E^{n}}$ be a payoff solution concept, where $2^{E^{n}}$ denotes the set of all subsets of the $n$-dimensional Euclidean space $E^{n}$. Intuitively, given that the players in $N$ align themselves into coalitions in the c.s. $P \in \Pi$, we interpret $S(P)$ as the set of all likely disbursements of payoffs to players in $N$. E.g. $S$ may denote the individually rational payoffs, the core, a $v N-M$ stable set, the Shapley value, the bargaining set $M_{1}^{(i)}$, the kernel, the nucleolus or any other payoff solution concept that indicates disbursement of payoffs as solutions of an $n$-person cooperative game. For $P \in \Pi$, $S(P)$ may be the empty set $\emptyset$ (e.g. the core), or a single point in $E^{n}$ (e.g. the Shapley value or the nucleolus) or a nonempty subset of $E^{n}$ (e.g. the bargaining set $M_{1}^{(i)}$ or the kernel). If $S(P)=\emptyset$ (interpreting this fact as players unable to reach an agreement on the disbursement of payoffs when they are aligned into coalitions in $P$ ), then we will assume for simplicity of exposition that $P$ is not viable. Let $\Pi(S)$ denote the set of all viable coalition structures with respect to the payoff solution concept (p.s.c.) $S$, i.e.

$$
\Pi(S)=\{P \in \Pi: S(P) \neq \emptyset\} \text {. }
$$

Definition 3.1. A solution configuration with respect to p.s.c. $S$ is a pair $(x, P)$ such that $x \in S(P)$ and $P \in \Pi(S)$.

A solution configuration w.r.t. p.s.c. $S$ represents a possible outcome of the $n$ person cooperative game where $S$ represents any appropriate payoff solution concept. Let $S C(S)$ denote the set of all solution configurations w.r.t. p.s.c. S, i.e.

$$
S C(S)=\underset{P \in \Pi(S)}{U}[S(P) \times\{P\}] .
$$

We now define a binary relation, domination, on the set $S C(S)$ as follows:

Definition 3.2. Let $\left(x, P_{1}\right)$ and $\left(y, P_{2}\right)$ belong to $S C(S)$. Then $\left(x, P_{1}\right)$ dominates $\left(y, P_{2}\right)$, denoted by $\left(x, P_{1}\right) \operatorname{dom}\left(y, P_{2}\right)$ iff

$\exists$ a nonempty $R \in P_{1}$ such that $x_{i}>y_{i}$ for all $i \in R$.

Intuitively, if $\left(x, P_{1}\right)$ dom $\left(y, P_{2}\right)$, then the players in some coalition $R$ in c.s. $P_{1}$ prefer $P_{1}$ to $P_{2}$. We require the players in subset $R$ to be together in a coalition in c.s. $P_{1}$ so that there is no conflict of interest between these player's preference for $P_{1}$ and their allegiance to the other players in their coalition.

The dominance relation as defined above may be neither asymmetric nor transitive. We now have an abstract game ( $S C(S)$,dom) where $S C(S)$ is the set of outcomes and dom is a binary relation on $S C(S)$. For this abstract game, we look at the core and the dynamic solution as defined in Section 2.

Definition 3.3. Let $\Gamma$ be an $n$-person cooperative game ${ }^{3}$ ) and $S$ be a p.s.c. The core of solution configurations w.r.t.p.s.c. $S$, denoted by $J_{0}(S)$, is the core of the abstract game (SC(S), dom).

Definition 3.4. Let $\Gamma$ be an $n$-person cooperative game and $S$ be a p.s.c. The dynamic

\footnotetext{
${ }^{3}$ ) In this section, $\Gamma$ denotes an $n$-person cooperative game with side payments, without side payments or a game in partition function form.
} 
solution of solution configurations w.r.t. p.s.c. $S$, denoted by $J_{1}(S)$, is the dynamic solution of the abstract game ( $S C(S)$,dom).

From Proposition 2.3, we obtain the following result.

Proposition 3.1. $J_{0}(S) \subset J_{1}(S)$.

The core of an abstract game is a very intuitive and plausible solution concept. However, for some games and for certain p.s.c., $J_{0}(S)$ may be an empty set. In such cases, we can proceed to look at $J_{1}(S)$ as a solution concept. If the p.s.c. $S$ is such that $S(P)$ is a unique point in $E^{n}$ for each $P \in \Pi(S)$ with $\Pi(S) \neq \emptyset$, then the set $S C(S)$ is finite and nonempty. By appealing to Theorem 2.5 , we conclude the following result.

Proposition 3.2. Let $\Gamma$ be an $n$-person cooperative game and $S$ be a p.s.c. such that $\Pi(S) \neq \emptyset$ and assume that $S(P)$ is a unique point in $E^{n}$ for each $P \in \Pi(S)$. Then $J_{1}(S) \neq \emptyset$.

In another approach, we model just the set of all viable coalition structures $\Pi(S)$ as the outcomes of an abstract game. A domination relation on $\Pi(S)$ is defined as follows.

Definition 3.5. Let $P_{1}, P_{2} \in \Pi(S), \emptyset \neq R \in 2^{N}$ and $S$ be a p.s.c. Then $P_{1}$ dominates $P_{2}$ via $R$ w.r.t. p.s.c. $S$, denoted by $P_{1} \operatorname{dom}_{R}(S) P_{2}$, iff

$$
R \in P_{1}
$$

and

$$
\text { for each } y \in S\left(P_{2}\right), \exists \text { an } x \in S\left(P_{1}\right) \text { such that } x_{i}>y_{i} \forall i \in R \text {. }
$$

Intuitively, if $P_{1} \operatorname{dom}_{R}(S) P_{2}$, then the players in subset $R$ prefer $P_{1}$ to $P_{2}$ because by Condition (3.5), no matter how the players disburse the payoffs corresponding to c.s. $P_{2}$, each player in $R$ will do better in c.s. $P_{1}$. Condition (3.4) is imposed for the same reasons Condition (3.3) is imposed in Definition 3.2.

Definition 3.6. Let $P_{1}, P_{2} \in \Pi(S)$ and $S$ be a p.s.c. $P_{1}$ dominates $P_{2}$ w.r.t. $S$, denoted by $P_{1} \operatorname{dom}(S) P_{2}$, iff

$$
\exists \text { a nonempty } R \in 2^{N} \text { such that } P_{1} \operatorname{dom}_{R}(S) P_{2} \text {. }
$$

We now have another abstract game.(I(S), $\operatorname{dom}(S))$ where $\Pi(S)$ is the set of outcomes and $\operatorname{dom}(S)$ is the binary relation on $\Pi(S)$. Once again we look at the core and the dynamic solution of this abstract game.

Definition 3.7. Let $\Gamma$ be an $n$-person cooperative game and $S$ be a p.s.c. The core of coalition structures w.r.t. p.s.c. $S$, denoted by $K_{0}(S)$, is the core of the abstract game $(\Pi(S), \operatorname{dom}(S))$.

Definition 3.8. Let $\Gamma$ be an $n$-person cooperative game and $S$ be a p.s.c. The dynamic solution of coalition structures w.r.t. p.s.c. $S$, denoted by $K_{1}(S)$, is the dynamic solution of the abstract game (ח(S), dom(S)).

Once again, by appealing to Proposition 2.3, we have: 
Proposition 3.3. $K_{0}(S) \subset K_{1}(S)$.

Also, since $\Pi(S)$ is always finite, we have:

Proposition 3.4. $K_{1}(S) \neq \emptyset$.

The following results gives a comparison of the two models.

Theorem 3.5. Let $\Gamma$ be an $n$-person cooperative game and $S$ be a p.s.c. Then we have

$$
K_{0}(S) \supset\left\{P \in \Pi:(x, P) \in J_{0}(S)\right\}
$$

Proof: Let $P_{1} \in\left\{P \in \Pi:(x, P) \in J_{0}(S)\right\}$. Then $\exists x \in S\left(P_{1}\right)$ such that $\left(x, P_{1}\right)$ is undominated in $S C(S)$ which implies that $P_{1}$ is undominated (w.r.t. $\left.S\right)$ in II(S), i.e., $P_{1} \in K_{0}(S)$.

Another consequence of the definitions of $K_{0}(S)$ and $J_{0}(S)$ is as follows:

Theorem 3.6. Let $\Gamma$ be an $n$-person cooperative game and $S$ be a.p.s.c. such that $\forall P \in \Pi, S(P)$ is either a single point set in $E^{n}$ or an empty set. Then

$$
K_{0}(S)=\left\{P \in \Pi:(x, P) \in J_{0}(S)\right\}
$$

and

$$
J_{0}(S)=\left\{(S(P), P): P \in K_{0}(S)\right\} .
$$

If $J_{0}(S) \neq \emptyset$, then the solution configuration model indicates both coalition structures and distribution of payoffs among the players as solution in $J_{0}(S)$ whereas the coalition structure model indicates only coalition structures as solutions in $K_{0}(S)$. Also by Theorem $3.5, J_{0}(S)$ indicates fewer (or at most an equal number of) coalition structures as solutions compared to $K_{0}(S)$. However, if the p.s.c. $S$ is such that for each $P \in \Pi, S(P)$ is either a single point in $E^{n}$ or an empty set, then the two models are identical (expect in form) and indicate the same results.

Note that the stability of the core is much stronger than the stability of the dynamic solution. Hence if $K_{0}(S)\left(J_{0}(S)\right)$ is non-empty, we shall use $K_{0}(S)\left(J_{0}(S)\right)$ as the prediction of the coalition structure (solution configuration) model. We shall look at $K_{1}(S)\left(J_{1}(S)\right)$ only if $K_{0}(S)\left(J_{0}(S)\right)$ is an empty set.

In the next section, the c.s. model is represented by means of digraphs. The predictions of this model, $K_{0}(S)$ and $K_{1}(S)$, are then described in graph theoretic terminology. Subsequently, in sections $5,6,7$ and 8 , the predictions of the c.s. model and the s.c. model w.r.t. various p.s.c.'s are studied for the special case of games with side payments.

\section{Representation by Digraphs}

Since the number of coalition structures is finite, we can represent the abstract game (II(S), dom(S)) of a game on $N$ by means of a directed graph (or digraph). Given a payoff solution concept $S$, let $D=D(S)$ be a digraph whose vertex set 
$V(D)=\Pi(S)$ and whose $\operatorname{arcset} A(D)$ is given by

$$
A(D)=\left\{\left(P_{1}, P_{2}\right) \in \Pi(S) \times \Pi(S): P_{1} \operatorname{dom}(S) P_{2}\right\}
$$

We call such a digraph $D$ the domination digraph of the abstract game (ח(S), dom(S)).

Example 4.1. Let $\Gamma$ be a 3-person game on $\{1,2,3\}$. Let $S$ be a p.s.c. defined as follows:

Let $0 \leqslant a \leqslant b \leqslant c=d$ be real numbers such that $c>a+b$ and

$$
S(P)=\left\{\begin{array}{l}
(0,0,0) \text { if } P=\{\{1\},\{2\},\{3\}\} \\
(0, a, 0) \text { if } P=\{\{1,2\},\{3\}\} \\
(0,0, b) \text { if } P=\{\{1,3\},\{2\}\} \\
\left\{\left(0, x_{2}, c-x_{2}\right): a \leqslant x_{2} \leqslant c-b\right\} \text { if } P=\{\{1\},\{2,3\}\} \text { or }\{\{1,2,3\}\} .
\end{array}\right.
$$

To condense notation, we shall drop the braces around coalitions in coalition structures and, for example, denote $\{\{1\},\{2,3\}\}$ by (1) (23). Note that

$$
\begin{aligned}
& \text { (1) (23) } \operatorname{dom}(S)(1)(2)(3), \\
& \text { (1) (23) } \operatorname{dom}(S)(12)(3), \\
& \text { (1) (23) } \operatorname{dom}(S)(13)(2)
\end{aligned}
$$

The domination graph of the game $\Gamma$ is shown in Figure 4.1.

Let $\left(P_{1}, P_{2}\right) \in A(D)$. Then we say $P_{1}$ is adjacent to $P_{2}$ and $P_{2}$ is adjacent from $P_{1}$. The outdegree, od $(P)$, for $P \in \Pi(S)$ is the number of c.s.'s adjacent from it and the indegree, $\mathrm{id}(P)$, for $P \in \Pi(S)$ is the number adjacent to it. Then, in terms of this terminology, the core of the abstract game $(\Pi(S)$, dom $(S))$ is given by

$$
K_{0}(S)=\{P \in V(D): \operatorname{id}(P)=0\}
$$

In Example 4.1, $K_{0}(S)=\{(1)(23),(123)\}$.

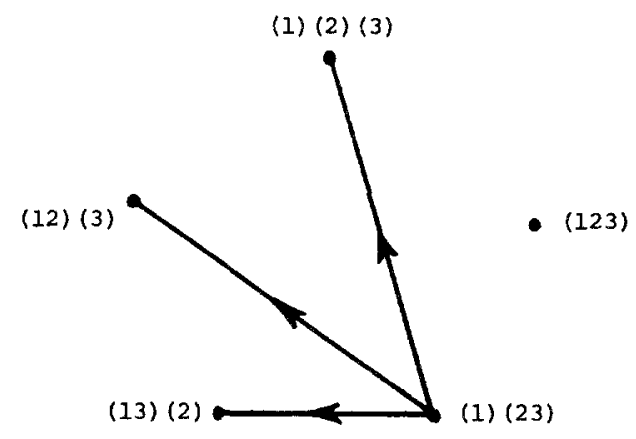

Fig. 4.1: The domination digraph of game in Example 4.1 
The converse digraph $D^{\prime}$ of $D$ has the same vertex set as $D$ and the arc $\left(P_{1}, P_{2}\right) \in A\left(D^{\prime}\right) \Leftrightarrow\left(P_{2}, P_{1}\right) \in A(D)$. Thus the converse of $D$ is obtained by reversing the direction of every arc in $D$. If $D=D(S)$ is the domination digraph of the abstract game $\left(\Pi(S)\right.$, dom $(S)$ ), then we call its converse $D^{\prime}=D^{\prime}(S)$ the transition digraph of the abstract game $(\Pi(S), \operatorname{dom}(S))$. The transition digraph of the game in Example 4.1 is shown in Figure 4.2

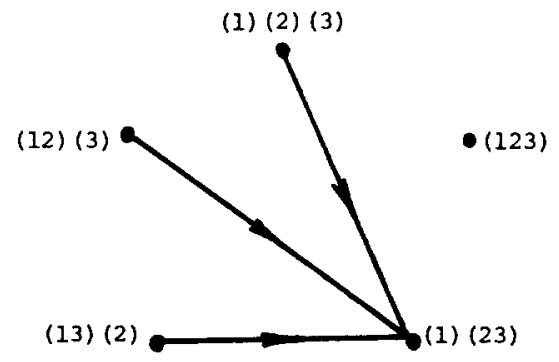

Fig. 4.2: The transition digraph of the game in Example 4.1

To define the dynamic solution in terms of the transition graph, we need a few basic definitions from graph theory [cf. Harary]. A (directed) walk in a digraph is an alternating sequence of vertices and $\operatorname{arcs} P_{0}, e_{1}, P_{1}, \ldots, e_{n}, P_{n}$ in which each arc $e_{i}$ is $\left(P_{i-1}, P_{i}\right)$. A closed walk has the same first and last vertex. A path is a walk in which all vertices are distinct; a cycle is a nontrivial closed walk with all vertices distinct (except the first and the last). If there is a path from $P_{1}$ to $P_{2}$, then $P_{2}$ is said to be accessible from $P_{1}$. A digraph is strongly connected or strong if any two vertices are mutually accessible. A strong component of a digraph is a maximal strong subgraph. Let $T_{1}, T_{2}, \ldots, T_{m}$ be the strong components of $D$. The condensation $D^{*}$ of $D$ has the strong components of $D$ as its vertices, with an arc from $T_{i}$ to $T_{j}$ whenever there is at least one arc in $D$ from a vertex of $T_{i}$ to a vertex of $T_{j}$. (See Figure 4.3). It follows from the maximality of strong components that the condensation $D^{*}$ of any graph $D$ has no cycles. Let $D^{\prime}(S)$ be the transition graph of the abstract game (II(S), $\left.\operatorname{dom}(S)\right)$ with strong components $T_{1}, T_{2}, \ldots, T_{m}$.
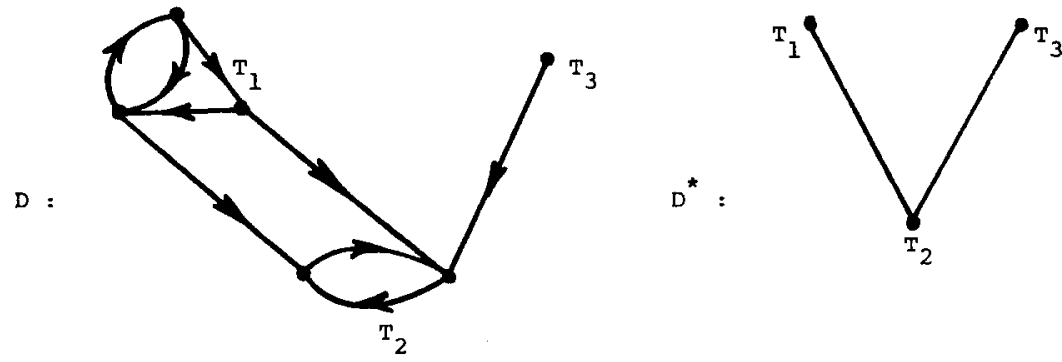

Fig. 4.3: A digraph and its condensation 
Then the dynamic solution of the abstract game is given by

$$
K_{1}(S)=\cup\left\{T_{i}: \operatorname{od}\left(T_{i}\right)=0 \text { in the condensation } D^{\prime *}\right\} .
$$

In Example 4.1, $K_{1}(S)=\{(1)(23),(123)\}$.

\section{Solutions with Respect to the Individually Rational Payoffs}

In the next four sections, we will characterize the solutions of the abstract games for the special case of games with side payments using various payoff solutions concepts.

A cooperative n-person game in characteristic function form with side payments is a pair $(N, v)$ where $N=\{1, \ldots, n\}$ denotes the set of players (as stated before) and $\nu$ is a nonnegative real-valued function defined on the subsets of $N$ which satisfies $v(\emptyset)=0$ and $\nu(\{i\})=0$ for $\left.^{4}\right)$ all $i \in N$.

The individually rational payoffs (i.r.p.) corresponding to coalition structure $P=\left(P_{1}, \ldots, P_{m}\right) \in \Pi$ is the set

and

$$
\Gamma(P)=\left\{x \in E^{n}: \sum_{i \in P_{j}} x_{i}=\nu\left(P_{j}\right) \text { for all } j=1, \ldots, m\right.
$$

$$
\left.x_{i} \geqslant v(i) \text { for }^{5} \text { ) all } i \in N\right\} \text {. }
$$

When $P=(N), I((N))$ is also referred to as the set of imputations. Since $I(P)$ is nonempty for all $P \in \Pi$, we have

$$
\Pi(I)=\Pi \text {. }
$$

A game $(N, v)$ is said to be superadditive if

$$
R_{1} \cap R_{2}=\emptyset, R_{1}, R_{2} \in 2^{N} \Rightarrow v\left(R_{1}\right)+v\left(R_{2}\right) \leqslant v\left(R_{1} \cup R_{2}\right)
$$

and strictly superadditive if strict inequality holds in Relation (5.1).

Define the worth of a coalition structure $P$ in the game $(N, v)$ by

$$
w(P)=\underset{P_{j} \in P}{\Sigma} v\left(P_{j}\right)
$$

Let

$$
z=\max _{P \in \Pi} w(P)
$$

${ }^{4}$ ) This condition and the nonnegativity restriction on $v$ causes no real loss of generality since all the payoff solution concepts we consider are invariant under strategic equivalence.

${ }^{5}$ ) To condense notation, we shorten expressions like $v(\{i, j, k\})$ to $v(i j k)$ and so on. 
and define

$$
\Pi_{z}=\{P \in \Pi: w(P)=z\} .
$$

If $x \in E^{n}$ and $R \subset N$, let $x(R)$ denote $\sum_{i \in R} x_{i}$. Then we have the following theorem.

Theorem 5.1. Let $\Gamma$ be an $n$-person cooperative game with side payments. Then $K_{0}(I) \neq \emptyset$. In particular, we have $K_{0}(I) \supset \Pi_{z}$.

Proof. Let $P^{1}=\left(P_{1}^{1}, P_{2}^{1}, \ldots, P_{m}^{1}\right) \in \Pi_{z}$. Suppose $\exists P \in \Pi$ such that $P \operatorname{dom}(I) P^{1}$, i.e.

$\exists R \in P$ such that $P \operatorname{dom}_{R}(I) P^{1}$. Now we can write $R=\bigcup_{i=1}^{m}\left(R \cap P_{i}^{1}\right)$. Pick $y \in I\left(P^{1}\right)$ such that $y\left(R \cap P_{i}^{1}\right)=v\left(P_{i}^{1}\right)$ if $R \cap P_{i}^{1} \neq \emptyset$ for all $i=1,2, \ldots, m$. Since $P \operatorname{dom}_{R}(I) P^{1}$, $\exists x \in I(P)$ s.t. $x_{i}>y_{i}$ for all $i \in R$. I.e. $v(R)=x(R)>y(R)=P_{i}^{1} \cap R \neq \emptyset\left(P_{i}^{1}\right)$. Pick $P^{2} \in \Pi$ as follows. $P^{2}=\{R\} \cup\left\{P_{i}^{1}: P_{\mathrm{i}}^{1} \cap R \Rightarrow \emptyset\right\} \cup\left\{P_{i}^{1}-R: P_{i}^{1} \cap R \neq \emptyset\right\}$ Then $w\left(P^{2}\right)>w\left(P^{1}\right)$, a contradiction! This completes the proof.

The following example will show that, in general, we cannot make a stronger statement than in the theorem above.

Example 5.1. Let $\Gamma$ be a 4-person game with $v(12)=v(34)=v(23)=1$, and $v(R)=0$ for all other $R \subset N$.

Let $P_{1}=(12)(34), P_{2}=(14)(23)$ and $P_{3}=(1)(23)(4)$. $w\left(P_{1}\right)=2$, $w\left(P_{2}\right)=w\left(P_{3}\right)=1$. But $K_{0}(I)=\left\{P_{1}, P_{2}, P_{3}\right\}$.

However, we do have the following.

Theorem 5.2. Let $\Gamma$ be an $n$-person game with side payments such that $(N) \in \Pi_{z}$. Then $K_{0}(I)=\Pi_{z}$.

Proof: From Theorem 5.1 we need prove only $K_{0}(I) \subset \Pi_{z}$. Let $P_{1} \in \Pi$ such that $P_{1} \notin \Pi_{z}$, i.e. $w\left(P_{1}\right)<z$. Then $(N) \operatorname{dom}(I) P_{1}$. This is seen as follows. Let $x \in I\left(P_{1}\right)$. Then $x(N)=w\left(P_{1}\right)<z$. Define $y$ so that $y_{i}=x_{i}+\left(z-w\left(P_{1}\right)\right) / n$ for all $i \in N$. Then $y \in I(\{N\})$ and $y_{i}>x_{i}$ for all $i \in N$.

Corollary 5.3. Let $\Gamma$ be a superadditive game. Then $K_{0}(I)=\Pi_{z}$. Furthermore, if $\Gamma$ is strictly superadditive, then $K_{0}(I)=\{(N)\}$.

Proof: $\Gamma$ superadditive $\Rightarrow(N) \in \Pi_{z}$, and $\Gamma$ strictly superadditive $\Rightarrow \Pi_{z}=\{(N)\}$.

For the solution configurations model, no general existence result is possible as is illustrated by the following example:

Example 5.2. Let $\Gamma=(N, v)$ be a 3-person game with

$$
v(12)=v(13)=v(23)=2, v(123)=2.5 \text {. }
$$

If can easily be shown that for this game $J_{0}(I)=\emptyset$. 


\section{Solutions with Respect to the Core}

Let $(N, v)$ be a cooperative game with side payments. Then the core of the game $(N, v)$ corresponding to c.s. $P \in \Pi$ is defined by

$$
\operatorname{Co}(P)=\left\{x \in I(P): x(R) \geqslant v(R) \text { for all } R \in 2^{N}\right\} .
$$

The core corresponding to a particular c.s. may be empty. Hence in general $\Pi(\mathrm{Co}) \neq \mathrm{I}$. in fact, for some games the core corresponding to every c.s. may be empty, i.e., $\Pi(\mathrm{Co})=\emptyset$. A characterization of $K_{0}(\mathrm{Co})$ and $J_{0}(\mathrm{Co})$ is as follows.

Theorem 6.1. Let $(N, v)$ be a cooperative game with side payments. Then,

$$
K_{0}(\mathrm{Co})=\Pi(\mathrm{Co})=\{P: \operatorname{Co}(P) \neq \emptyset\} .
$$

Also

$$
J_{0}(\mathrm{Co})=S C(\mathrm{Co})=\underset{P \in \Pi(\mathrm{Co})}{U}[\mathrm{Co}(P) \times\{\mathrm{P}\}]
$$

Proof: Let $P_{1}, P_{2} \in \Pi(\mathrm{Co})$. Suppose $P_{1} \operatorname{dom}_{R}(\mathrm{Co}) P_{2}$ for some $R \in P_{1}$. Let $y \in \operatorname{Co}\left(P_{2}\right)$. Then $\exists x \in \operatorname{Co}\left(P_{1}\right)$ s.t. $x_{i}>y_{i}$ for all $i \in R$. I.e. $x(R)>y(R)$. But since $R \in P_{1}$, $x(R)=v(R)$. Hence $y(R)<v(R)$ contradicting the fact that $y \in \operatorname{Co}\left(P_{2}\right)$. The proof of the second assertion is similar to the first.

Corollary 6.2. Let $(N, v)$ be a cooperative game with side payments. Let $S$ be a p.s.c. such that, for all $P \in \Pi, S(P) \subset I(P)$, and $S(P) \cap \operatorname{Co}(P) \neq \emptyset$ whenever $\operatorname{Co}(P) \neq \emptyset$. Then $K_{0}(\mathrm{Co}) \subset K_{0}(S)$ and $\left\{\mathrm{P}:(x, P) \in J_{0}(\mathrm{Co})\right\} \subset\left\{\mathrm{P}:(x, P) \in J_{0}(S)\right\}$.

In light of Theorem 6.1 we would like to characterize the coalition structures with nonempty cores. The next two theorems along with a known characterization of games with nonempty cores corresponding to the grand coalition $N$ accomplish this task.

Theorem 6.3. Let $(N, v)$ be a cooperative game with side payments. If $\Pi(\mathrm{Co}) \neq \emptyset$, then $\Pi(\mathrm{Co})=\Pi_{z}$.

Proof: Let $P_{1} \in \Pi(\mathrm{Co})$, and suppose $P_{1} \notin I_{z}$. Then $\exists P_{2} \in \Pi$ such that $w\left(P_{2}\right)>w\left(P_{1}\right)$. Let $x \in \operatorname{Co}\left(P_{1}\right)$. Then $x(R) \geqslant v(R)$ for all $R \subset N$ which implies that $w\left(P_{1}\right)=x(N) \geqslant w\left(P_{2}\right)$ and this is a contradiction! Hence $\Pi(\mathrm{Co}) \subset \Pi_{z}$.

Let $P_{1} \in \Pi_{z}$ and assume $P_{2} \in \Pi(\mathrm{Co}) \subset \Pi_{z}$. Let $x \in \operatorname{Co}\left(P_{2}\right)$. Then $x(R) \geqslant v(R)$ for all $R \subset N$. If $x(P)>v(P)$ for some $P \in P_{1}$, then $w\left(P_{2}\right)=x(N)>w\left(P_{1}\right)$, contradicting the fact that $P_{1} \in \Pi_{z}$. Hence $x(P)=v(P)$ for all $P \in P_{1} \Rightarrow x \in \operatorname{Co}\left(P_{1}\right) \Rightarrow P_{1} \in \Pi(\mathrm{Co})$. Therefore $\Pi(\mathrm{Co}) \supset \Pi_{z}$.

Corollary 6.4: Let $(N, v)$ be a game with side payments. Then for all $P_{1}, P_{2} \in \Pi(\mathrm{Co})$, $\operatorname{Co}\left(P_{1}\right)=\operatorname{Co}\left(P_{2}\right)$. 
Corollary 6.5. Let $(N, v)$ be a game with side payments. If there is a $P \in \Pi_{z}$ such that $\operatorname{Co}(P)=\emptyset$, then $\Pi(\mathrm{Co})=\emptyset$.

Given a game $\Gamma=(N, v)$ define game $\Gamma_{z}=\left(N, v_{z}\right)$ derived from $\Gamma$ as follows.

$$
v_{z}(R)=\left\{\begin{array}{l}
z \text { if } R=N \\
v(R) \text { for all other } R \subset N
\end{array}\right.
$$

$$
\text { where } z=\max _{P \in \Pi} w(P) \text {. }
$$

When there is more than one game under discussion, we shall denote the sets $\operatorname{Co}(P), \Pi(\mathrm{Co})$ and $\Pi_{z}$ by $\operatorname{Co}(P, \Gamma), \Pi(\operatorname{Co}, \Gamma)$, and $\Pi_{z}(\Gamma)$, respectively.

Theorem 6.6. Let $\Gamma=(N, v)$ be a game and $\Gamma_{z}$ be as in Relation (6.2) Then if $\operatorname{Co}(P, \Gamma) \neq \emptyset, \operatorname{Co}(P, \Gamma)=\operatorname{Co}\left((N), \Gamma_{z}\right)$.

Proof: From the definition of $\Gamma_{z}$ it is clear that for $P \neq(N) \operatorname{Co}(P, \Gamma)=\operatorname{Co}\left(P, \Gamma_{z}\right)$. From Theorem 6.3 we obtain $\Pi\left(\mathrm{Co}, \Gamma_{z}\right)=\Pi_{z}\left(\Gamma_{z}\right)$. Since $(N) \in \Pi_{z}\left(\Gamma_{z}\right)$, by Corollary 6.4 , $\operatorname{Co}\left(P, \Gamma_{z}\right)=\operatorname{Co}\left((N), \Gamma_{z}\right)$. Hence the theorem follows.

Games with nonempty cores corresponding to the grand coalition have been characterized by Bondareva [1962, 1963] and Shapley [1967]. For the sake to completeness we will repeat this characterization here.

A balanced set $B$ is defined to be a collection of subsets $R$ of $N$ with the property that there exist positive numbers $\delta_{R} \forall R \in B$ called weights, such that for each $i \in N$ we have

$$
\sum_{\{R \in B: i \in R\}} \delta_{R}=1
$$

A game $(N, v)$ is called balanced iff

$$
\sum_{R \in B} \delta_{R} v(R) \leqslant v(N)
$$

holds for every balanced set with weights $\left\{\delta_{R}\right\}$. The following theorem was proved by Bondareva [1962, 1963] and Shapley [1967].

Theorem 6.7. Let $(N, v)$ be a game. Then $\operatorname{Co}((N)) \neq \emptyset$ if and only if the game is balanced.

Corollary 6.8. Let $\Gamma=(N, v)$ be a game. Then $\Pi(\mathrm{Co}, \Gamma) \neq \emptyset$ if and only if the game $\left(N, v_{z}\right)$ is balanced.

Proof: (Necessity): $\Pi(\operatorname{Co}, \Gamma) \neq \emptyset \Rightarrow \operatorname{Co}\left((N),\left(N, v_{z}\right)\right) \neq \emptyset$ (by Theorem 6.6$) \Rightarrow\left(N, v_{z}\right)$ is balanced (by Theorem 6.7). 
(Sufficiency): If $\Gamma_{z}=\left(N, v_{z}\right)$ is balanced $\Rightarrow \operatorname{Co}\left((N), \Gamma_{z}\right) \neq \emptyset$ (by Theorem 6.7). If $(N) \in \Pi_{z}(\Gamma)$ then $\Gamma=\Gamma_{z}$ and we are finished.Otherwise $\exists P \in \Pi_{z}\left(\Gamma_{z}\right)$ such that $P \neq(N)$. Then, $\operatorname{Co}(P, \Gamma)=\operatorname{Co}\left(P, \Gamma_{z}\right)=\operatorname{Co}\left((N), \Gamma_{z}\right) \neq \emptyset$.

Thus we have completely characterized $K_{0}(\mathrm{Co})$ and $J_{0}(\mathrm{Co})$ for all games with side payments.

Example 6.1. (A game with no solution. See Lucas [1968, 1969].) Let

$N=\{1,2,3,4,5,6,7,8,9,10\}$ and $v$ be given by

$$
\begin{aligned}
& v(N)=5, v(13579)=4, \\
& v(12)=v(34)=v(56)=v(78)=v(910)=1, \\
& v(3579)=v(1579)=v(1379)=3, \\
& v(357)=v(157)=v(137)=2, \\
& v(359)=v(159)=v(139)=2, \\
& v(1479)=v(3679)=v(5279)=2, \text { and } \\
& v(R)=0 \text { for all other } R \subset N .
\end{aligned}
$$

In this game $z=5, \Pi_{z}=\left\{(N), P_{1}=(12)(34)(56)(78)(910)\right\}$ and $\operatorname{Co}((N))=\operatorname{Co}\left(P_{1}\right)=\{x: x(12)=x(34)=x(56)=x(78)=x(910)=1$, and $x(13579) \geqslant 4$ \}. By Theorem 6.1 ,

$$
K_{0}(\mathrm{Co})=\left\{(N), P_{1}\right\}
$$

and

$$
J_{0}(\mathrm{Co})=\operatorname{Co}((N)) \times\left\{(N), P_{1}\right\}
$$

\section{Solutions with Respect to the Shapley Value}

Shapley [1953] defined a unique value satisfying three axioms for all $n$-person cooperative games with side payments. It was assumed that the grand coalition would form. Later, Aumann/Dreze [1974] generalized the axioms to define the Shapley value for all coalition structures.

A permutation $\alpha$ of $N$ is a one-one function from $N$ onto itself. For $R \in 2^{N}$, write $\alpha R=\{\alpha i: i \in R\}$. If $v$ is a game on $N$, define a game $\alpha * v$ on $N$ by

$$
(\alpha * \nu)(R)=v(\alpha R) \text { for all } R \in 2^{N} \text {. }
$$

Also, if $v$ and $u$ are games on $N$, define a game $v+u$ on $N$ by

$$
(v+u)(R)=v(R)+u(R) \text { for all } R \in 2^{N} \text {. }
$$

Call a c.s. $P=\left(P_{1}, \ldots, P_{m}\right)$ invariant under $\alpha$ if $\alpha P_{j}=P_{j}$ for all $j=1, \ldots, m$. Player $i$ is null if $v(R \cup(i))=v(R)$ for all $R \in 2^{N}$. Let $G^{N}$ denote the set of all games with side payments on $N$. Since we assume that for all games with side payments, $v(\emptyset)=0$ and $v(i)=0 \forall i \in N, G^{N}$ is a Euclidean space of dimension $2^{n}-(n+1)$. 
Fix $N=\{1, \ldots, n\}$ and $P=\left(P_{1}, \ldots, P_{m}\right) \in \Pi$. The Shapley value corresponding to c.s. $P$ is a function $\Phi_{P}$ from $G^{N}$ to $E^{n}$ i.e. a function that associates with each game a payoff vector satisfying the following axioms:

A.1 (Relative Efficiency): $\Phi p(v)\left(P_{j}\right)=v\left(P_{j}\right)$ for all $j=1, \ldots, m$.

A.2 (Symmetry): For all permutations $\alpha$ of $N$ under which $P$ is invariant,

$$
\Phi_{p}(\alpha * v)(R)=\Phi_{p}(v)(\alpha R) .
$$

A.3 (Additivity): $\Phi_{p}(v+u)=\Phi_{p}(v)+\Phi_{p}(u)$.

A.4 (Null Player Axiom): If $i$ is a null player, then $\Phi p(v)(i)=0$.

When $P=(N)$, the above axioms are equivalent to Shapley's axiom which specify a unique value $\phi(v)=\left(\phi_{1}(v), \ldots, \phi_{n}(v)\right)$ given by

$$
\phi_{i}(v)=\Phi_{(N)}(v)(i)=\sum_{R \subset N} \frac{(r-1) !(n-r) !}{n !}[v(R)-v(R-\{i\})]
$$

where $r=|R|$, the cardinality of coalition $R$. For each $R \in 2^{N}$, denote by $\nu \mid R$ the game on $R$ defined for all $T \subset R$ by

$$
(v \mid R)(T)=v(T) \text {. }
$$

Theorem 7.1. Fix $N$ and $P=\left(P_{1}, \ldots, P_{m}\right)$. Then there is a unique value $\Phi_{p}$ and it is given for all $j=1, \ldots, m$ and $i \in P_{j}$ by

$$
\left(\Phi_{P} v\right)(i)=\left(\Phi_{\left(P_{j}\right)}\left(v \mid P_{j}\right)\right)(i) \text {. }
$$

Proof: See Aumann/Dreze [1974, 220-221].

Since $\Phi(P)$ is $\left.{ }^{6}\right)$ nonempty for all $P \in \Pi, \Pi(\Phi)=\Pi$. Also note from (7.3) that if $v$ is superadditive, then $\Phi(P)(i) \geqslant 0$ and hence $\Phi(P) \in I(P)$. Also, since $\Phi(P)$ consists of a unique outcome for all $P \in \Pi$, by Theorem 3.6 the s.c. model and the c.s. model give identical results. For convenience, all the results in this section are stated only for the c.s. model.

A partial existence theorem for $K_{0}(\Phi)$ is as follows:

Theorem 7.2. Let $\Gamma$ be an $n$-person game in which the only coalitions with positive values are all the $(n-1)$-person and $n$-person coalitions. Then $K_{0}(\Phi) \neq \emptyset$.

Proof: Let us denote the game as follows:

$$
\begin{aligned}
& v(i)=0 \text { for all } i \in N, \\
& v(N-(i))=a_{i} \text { for all } i \in N, \\
& v(N)=b, \text { and } v(R)=0 \text { for all other } R \subset N .
\end{aligned}
$$

$\left.{ }^{6}\right)$ When there is no doubt about the game $v$ under consideration, we shall denote $\Phi$ p $(v)$ by $\Phi(P)$ which is consistent with the previous section. 
We can assume (by relabelling of the players) that

$$
a_{1} \leqslant a_{2} \leqslant \ldots \leqslant a_{n}
$$

Let $a=\sum_{i=1}^{n} a_{i}$ and $\Pi_{a_{n}}=\left\{P \in \Pi: w(P)=a_{n}\right\}$. Using (7.3) and (7.5) we have

$$
\Phi((N))(i)=\left((n-1) b+a-n \cdot a_{i}\right) /(n(n-1)) .
$$

By (7.6) we have

$$
\Phi((N))(1) \geqslant \Phi((N))(2) \geqslant \ldots \geqslant \Phi((N))(n) .
$$

Also

$$
\Phi((N-i)(i))(j)= \begin{cases}a_{i} /(n-1) & \text { for } j=1, \ldots, n \\ & j \neq i \\ 0 & \text { for } j=i .\end{cases}
$$

Clearly, the only c.s.'s we need look at are $(N)$ and $(N-i)(i)$ for $i=1, \ldots, n$. All the c.s.'s not in $\Pi_{a_{n}}$ (except $\left.(N)\right)$ are dominated by c.s.'s in $\Pi_{a_{n}}$. From Expressions (7.7), (7.7), (7.8) and (7.9) it follows that $(N) \operatorname{dom}(\Phi)(N-n)(n)$ iff

$$
\Phi((N))(n-1)>\Phi((N-n)(n))(n-1)
$$

i.e.iff

$$
b>\left(n\left(a_{n}+a_{n-1}\right)-a\right) /(n-1) .
$$

Also if $a_{n}=a_{n-1}$ (i.e. $\left.(N-(n-1))(n-1) \in \Pi_{a_{n}}\right)$ then

$$
(N) \operatorname{dom}(\Phi)(N-(n-1))(n-1)
$$

iff

$$
\Phi((N))(n)>\Phi((N-(n-1))(n-1))(n),
$$

i.e. iff

$$
b>\left(n\left(a_{n}+a_{n-1}\right)-a\right) /(n-1) .
$$

Now,

$$
(N-n)(n) \operatorname{dom}(\Phi)(N)
$$

iff

$$
\Phi((N-n)(n))(1)>\phi((N))(1),
$$

i.e. iff

$$
b<\left(n\left(a_{n}+a_{1}\right)-a\right) /(n-1) .
$$


Hence we have

$$
K_{0}(\Phi)= \begin{cases}(N) & \text { If } b>\left(n\left(a_{n}+a_{n-1}\right)-a\right) /(n-1) \\ \Pi_{a_{n}} & \text { if } \mathrm{b}<\left(n\left(a_{n}+a_{1}\right)-a\right) /(n-1) \\ (N) \cup \Pi_{a_{n}} & \text { otherwise. }\end{cases}
$$

Corollary 7.3. Let $\Gamma$ be a 3-person game with side payments. Then $K_{0}(\Phi) \neq \emptyset$.

In general, this is the strongest existence result we can obtain. I.e. there is a 4-person game for which $K_{0}(\Phi)=\emptyset$. This is shown in Example 7.5.

If $\operatorname{Co}(P) \neq \emptyset, \Phi(P)$ may not belong to $\operatorname{Co}(P)$. Hence Corollary 6.2 is not applicable for the Shapley value. The following example illustrates this fact.

Example 7.1. Let $N=\{1,2,3\}$ and $v$ be given by $v(1)=v(2)=v(3)=0, v(12)=50$, $v(13)=50, v(13)=56$, and $v(123)=80$. Then the Shapley value is given by:

$$
\Phi(P)= \begin{cases}(24.67,27.67,27.67) & \text { if } P=(123) \\ (0,28,28) & \text { if } P=(1)(23) \\ (25,0,25) & \text { if } P=(13)(2) \\ (25,25,0) & \text { if } P=(12)(3) \\ (0,0,0) & \text { if } P=(1)(2)(3)\end{cases}
$$

Note that $\operatorname{Co}((123))=\operatorname{Conv}\{(20,30,30),(24,26,30),(24,30,26)\}$ but $\Phi((123)) \notin \operatorname{Co}((123))$. The transition digraph is shown in Figure 7.1, and hence $K_{0}(\Phi)=K_{1}(\Phi)=(1)(23)$.

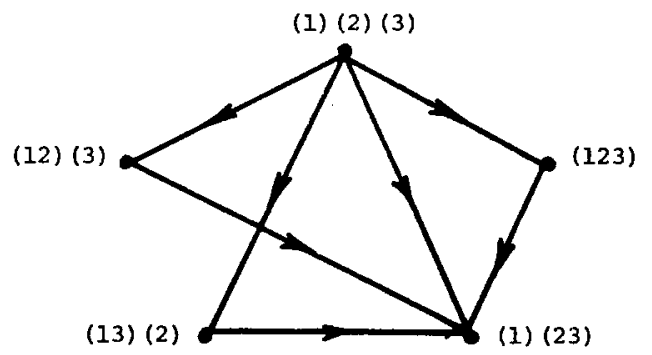

Fig. 7.1: The transition digraph for Example 7.1

The above example illustrates a weakness of the Shapley value in that the Shapley value is derived entirely from the characteristic function rather than the bargaining positions of the players in the process of coalition formation. However, the Shapley value has been extensively used as an a priori measure of power of players in "simple" games. Hence the study of $K_{0}(\Phi)$ and $K_{1}(\Phi)$ is most appropriate for simple games.

The class of all simple games forms a subclass of the class of all cooperative games with side payments. A simple game is a game in which every coalition has value either 1 or 0 . A coalition $R \subset N$ is winning if $v(R)=1$ and losing if $v(R)=0$. A simple game 
can be represented by a pair $(N, W)$ where $N$ is the set of players and $W$ is the set of winning coalitions. A simple game is monotonic iff $R \in W$ and $T \supset R \Rightarrow T \in W$, and proper iff $R \in W \Rightarrow N-R \notin W$. A winning coalition $R$ is called minimal winning if every proper subset of $R$ is losing. A monotonic simple game can be represented by the pair $\left(N, W^{m}\right)$ where $W^{m}$ is the set of all minimal winning coalitions. If $W^{m}=\{\{i\}\}$, then player $i$ is said to be a dictator. If $j \in \cap \omega^{m} \neq \emptyset$, then player $j$ is said to be a veto player. If $k \notin \cup W^{m}$ then player $k$ is said to be a dummy. Dummies play no active role in the game and for all practical purposes can be omitted from the set of players. A weighted majority game is a monotonic simple game that can be represented by

$$
\left[q: a_{1}, a_{2}, \ldots, a_{n}\right]
$$

where $q \geqslant 0$ is called the quota, $a_{i} \geqslant 0, i=1, \ldots, n$ is the weight of the $i$-th player, and $R \in W \Leftrightarrow \sum_{i \in R} a_{i} \geqslant q$. Expression (7.10) is said to be a weighted majority representation of the simple game. Two weighted majority representations are said to be equivalent if they represent the same simple game. E.g. $[2 ; 1,1,1]$ and $[5 ; 2,3,4]$ are equivalent since both represent the game $\left(\{1,2,3\}, W^{m}=\{(12),(13),(23)\}\right)$. Not every monotonic simple game has a weighted majority representation [see Shapley, 1962].

Example 7.2. The most common of all simple games is the straight majority game $M_{n}, n$ odd, in which

$$
W^{m}=\{R \subset N:|R|=(n+1) / 2\}
$$

where $|R|$ denotes the cardinality of coalition $R$. The Shapley value is given by

$$
\Phi(P)(i)= \begin{cases}1 /|R| & \text { if } i \in R \in W, R \in P \\ 0 & \text { otherwise. }\end{cases}
$$

It is clear that

$$
K_{1}(\Phi)=K_{0}(\Phi)=\{P \in \Pi: P \text { contains a minimal winning coalition }\} .
$$

Example 7.3. The pure bargaining game $B_{n}$, is given by $\omega^{m}=\{(N)\}$. The Shapley value is given by

$$
\Phi(P)(i)= \begin{cases}1 / n & \text { if } P=(N) \\ 0^{*} & \text { otherwise }\end{cases}
$$

clearly, $K_{1}(\Phi)=K_{0}(\Phi)=\{(N)\}$.

Example 7.4. Let $\Gamma$ be a proper game with a dictator. Then

$$
\Phi(P)(i)= \begin{cases}1 & \text { if } i \text { is a dictator } \\ 0 & \text { otherwise }\end{cases}
$$


Hence $K_{1}(\Phi)=K_{0}(\Phi)=\Pi$. Note that every player who is not a dictator is a dummy. So essentially we have a 1 -person game in which the only player is winning by himself.

Example 7.5. Consider the weighted majority game $[3 ; 2,1,1,1]$. The minimal winning coalitions are $W^{m}=\{(12),(13),(14),(234)\}$. The Shapley value is given by

$$
\Phi(P)= \begin{cases}(1 / 2,1 / 6,1 / 6,1 / 6) & \text { if } P=(1234) \\ (2 / 3,1 / 6,1 / 6,0) & \text { if } P=(123)(4) \\ (2 / 3,1 / 6,0,1 / 6) & \text { if } P=(124)(3) \\ (2 / 3,0,1 / 6,1 / 6) & \text { if } P=(134)(2) \\ (1 / 2,1 / 2,0,0) & \text { if } P=(12)(34) \text { or }(12)(3)(4) \\ (1 / 2,0,1 / 2,0) & \text { if } P=(13)(24) \text { or }(13)(2)(4) \\ (1 / 2,0,0,1 / 2) & \text { if } P=(14)(23) \text { or }(14)(2)(3) \\ (0,1 / 3,1 / 3,1 / 3) & \text { if } P=(1)(234) \\ (0,0,0,0) & \text { otherwise. }\end{cases}
$$

The transition digraph of the game is shown in Figure 7.2. Since all c.s.'s that contain only losing coalitions are dominated, these are omitted from this transition digraph. Note that $K_{0}(\Phi)=\emptyset$. However,

$$
\begin{aligned}
K_{1}(\Phi)= & \{(1)(234),(12)(3)(4),(12)(34),(134)(2),(13)(24) \\
& (13)(2)(4),(124)(3),(14)(23),(14)(2)(3),(123)(4)\} .
\end{aligned}
$$

A closer look at the Shapley value for different c.s.'s in Example 7.5 reveals the following observation. If players 1 and 2 who are in a winning coalition with 3 in the c.s. (123) (4) decide to expel player 3 from the coalition and form the smaller winning coalition (12), one would expect both players not to decrease their power in the smaller winning coalition (12) since there are fewer players to share the same amount of power. However, player 1 actually does decrease his power from $2 / 3$ to $1 / 2$. We shall call this phenomenon the paradox of smaller coalitions. To understand why this phenomenon occurs, let us look at Theorem 7.1. It states that given a c.s.

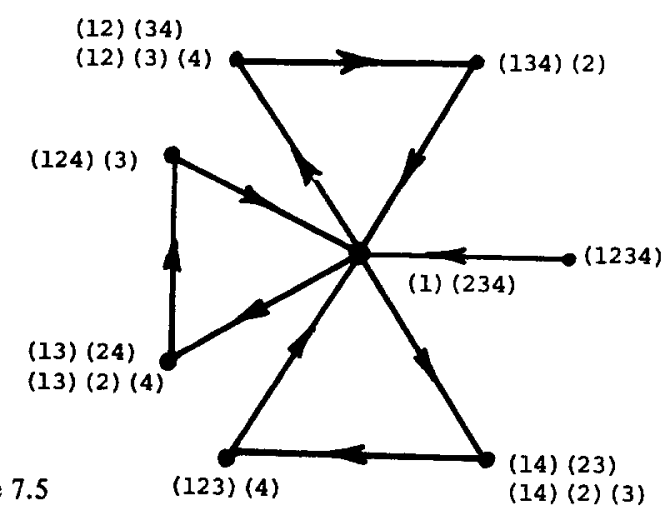

Fig. 7.2: The transition digraph in Example 7.5 
$P=\left(P_{1}, \ldots, P_{m}\right)$ the Shapley value of player $i$ in coalition $P_{j}$ depends only on the subgame $v\left\{P_{j}\right.$. I.e. the Shapley value of a player in a coalition is oblivious of the presence of other players not in the coalition for bargaining purposes. We shall regard this phenomenon as a "flaw" in the properties of the Shapley value. To make the above discussion more formal, let $\Gamma=(N, W)$ be a simple game and $\sigma$ be a payoff value concept (i.e. for all games and for each $P \in \Pi, \sigma(P)$ is a single point in $E^{n}$, where $n=$ the number of players). We say $\Gamma$ does not exhibit the paradox of smaller coalitions w.r.t. payoff value concept $\sigma$ iff the following holds:

Let $P_{1}, P_{2} \in \Pi$ such that there exists $P_{1} \in P_{1} \cap W$ and $P_{2} \in P_{2} \cap W$ satisfying $P_{2} \subset P_{1}$. Then $\sigma\left(P_{2}\right)(i) \geqslant \sigma\left(P_{1}\right)(i)$ for all $i \in P_{2}$.

The following result is a consequence of the above definition.

Theorem 7.4. Let $\Gamma$ be a proper monotonic simple game that does not exhibit the paradox of smaller coalitions w.r.t. $\Phi$. Then $K_{0}(\Phi) \neq \emptyset$.

Proof: Let $T \in W^{m}$ such that $|T| \leqslant|R|$ for all $R \in W^{m}$. Let $P \in \Pi$ be such that $T \in P$. Then $\Phi(P)(i)=1 /|T|$ for all $i \in T$. Suppose $\exists P_{1} \in \Pi$ such that $P_{1} \operatorname{dom}_{R}(\Phi) P$ for some $R \in P_{1}$, i.e., $\Phi\left(P_{1}\right)(i)>\Phi(P)(i)$ for all $i \in R$. Let $R^{\prime}$ be any minimal winning coalition contained in $R$, i.e. $R^{\prime} \subset R$ and $R^{\prime} \in W^{m}$. Let $P_{2} \in \Pi$ be such that $R^{\prime} \in P_{2}$. Then since $\Gamma$ does not exhibit the paradox, $\Phi\left(P_{2}\right)(i) \geqslant \Phi\left(P_{1}\right)(i)$ for all $i \in R^{\prime}$. Also

$$
\Phi\left(P_{2}\right)(i)= \begin{cases}1 /\left|R^{\prime}\right| & \text { if } i \in R^{\prime} \\ 0 & \text { otherwise. }\end{cases}
$$

Since $\Gamma$ is proper, $R^{\prime} \cap T \neq \emptyset$. Hence for all $i \in R^{\prime} \cap T$, $1 /\left|R^{\prime}\right|=\Phi\left(P_{2}\right)(i) \geqslant \Phi\left(P_{1}\right)(i)>\Phi(P)(i)=1 /|T|$, which is a contradiction (since $\left.\left|R^{\prime}\right| \geqslant|T|\right)$ !

Let

$$
t=\min _{R \in W^{m}}|R|
$$

and let

$$
\Pi_{t}=\{P \in \Pi: P \text { contains a winning coalition of size } t\} .
$$

Then we obtain the following.

Corollary 7.5. Let $\Gamma$ be a proper monotonic simple game that does not exhibit the paradox of smaller coalitions w.r.t. $\Phi$. Then $K_{0}(\Phi) \supset \Pi_{t}$.

That in general we cannot strengthen the above result is shown by the following example. 
Example 7.6. Let $\Gamma$ be a 4-person game represented by $[4 ; 2,2,1,1]$. The minimal winning coalitions are $\{(12),(34),(234)\}$. The Shapley value is given by

$$
\Phi(P)= \begin{cases}(1 / 2,1 / 2,0,0) & \text { if } P=(12)(34) \text { or (12) (3) (4) } \\ (1 / 2,1 / 2,0,0) & \text { if } P=(123)(4) \text { or }(124)(3) \\ (1 / 3,0,1 / 3,1 / 3) & \text { if } P=(134)(2) \\ (0,1 / 3,1 / 3,1 / 3) & \text { if } P=(1)(234) \\ (1 / 3,1 / 3,1 / 6,1 / 6) & \text { if } P=(1234)\end{cases}
$$

Note that the game does not exhibit the paradox of smaller coalitions. Also $t=2$, and $\Pi_{t}=\{(12)(3)(4),(12)(34)\}$. However, $K_{0}(\Phi)=\{(12)(3)(4),(12)(34),(123)(4)$, (124) (3)\}. Observe that players 3 and 4 are dummies in the subgame on $\{1,2,3\}$ and $\{1,2,4\}$ respectively.

An interesting problem raised by Theorem 7.4 is to characterize the class of games that do not exhibit the paradox of smaller coalitions w.r.t. $\Phi$. Let us look at symmetric games. A game $(N, v)$ is called symmetric if the value of a coalition depends only on the size of the coalition. A symmetric monotonic simple game is of the type $M_{n, k}=(N, W)$ where $W=\{R \subset N:|R| \geqslant k\}$. The following proposition follows from the symmetry axiom of the Shapley value.

Proposition 7.6. Let $\Gamma$ be a symmetric monotonic simple game. Then $\Gamma$ does not exhibit the paradox of smaller coalitions w.r.t. $\Phi$. In fact, $K_{0}(\Phi)=\Pi_{t}$.

Proof: The Shapley value is given by

$$
\Phi(P)(i)= \begin{cases}1 /|R| & \text { if } i \in R \in P \text { and } R \in W \\ 0 & \text { otherwise. }\end{cases}
$$

Hence the result follows from Statement (7.5).

The result in Proposition 7.6 (and also in Example 7.2) is known as "Riker's size principle" in the political science literature [see Riker/Ordeshook]. Riker's theory is that in zero-sum games with side payments, only minimal winning coalitions will form. Note that $\Pi_{t}$ consists of all minimal winning coalitions only. The result in Example 7.6 shows that the size principle does not generalize further.

Since Example 7.6 does not exhibit the paradox and is not symmetric, Proposition 7.6 is not a complete characterization. A list of all proper simple games with four or fewer players is given in the appendix along with the Shapley value $\Phi$ corresponding to all coalition structures, $K_{0}(\Phi)$, and whether or not the game exhibits the paradox.

Another interesting problem is to determine, if possible, a power index that has all the desirable properties of the Shapley value but that does not exhibit the paradox of smaller coalitions. 
The most critical axiom of the Aumann-Dreze generalization of the Shapley value is A.3.

$$
\text { A.3. } \Phi_{p}(v+u)=\Phi_{p}(v)+\Phi_{p}(u) \text {. }
$$

This axiom is acceptable if and only if we assume that the c.s. $P$ is fixed and that players in a coalition $P_{k} \in P$ cannot bargain on the basis of the value of coalitions not contained in $P_{k}$. This assumption is not appropriate for our model where the players are bargaining for a coalition structure and no c.s. is fixed.

Another generalization of the Shapley value (which he defined only for the grand coalition) to the case of all coalition structures which is appropriate for monotonic simple games is as follows.

(i) The Shapley value corresponding to the grand coalition is used as an a priori measure of power of the players. This is suggested by Shapley/Shubik [1954].

(ii) And within any coalition in a c.s., a player can expect to share in the payoff proportional to his power as defined in (i). This is suggested by Gamson [1961]. Assumptions (i) and (ii) define a unique value for all monotonic simple games which we denote by $\Phi^{\prime}$.

The (generalized) Shapley value $\Phi^{\prime}$ is a function from $\Pi \times G^{N}$ to $E^{n}$, i.e., a function that associates with each game and a c.s. a payoff vector given by Expression (7.3) and

$$
\Phi^{\prime}(P, v)(j)= \begin{cases}\frac{\phi_{j}(v)}{\sum_{i \in P_{k}} \phi_{i}(v)} \cdot v\left(P_{k}\right) \text { where } P_{k} \in P \text { is such that } \\ & j \in P_{k}, \text { if } j \text { is not a null player } \\ 0 & \text { if } j \text { is a null player }\end{cases}
$$

Note that $\Phi^{\prime}((N), v)=\Phi_{(N)}(v)=\phi(v)=\left(\phi_{1}(v), \ldots, \phi_{n}(v)\right)$. A simple consequence of the definition of $\Phi^{\prime}$ is as follows.

Proposition 7.7. Let $\Gamma$ be a monotonic simple game. The $\Gamma$ does not exhibit the paradox of smaller coalitions w.r.t. $\Phi^{\prime}$.

Proof: This follows from Expression (7.13).

In view of Corollary 7.5 , we might be tempted to assert that $K_{0}\left(\Phi^{\prime}\right) \supset \Pi_{t}$. However, the following example shows that it is not true.

Example 7.7. Consider the weighted majority game given in Example $7.5[3 ; 2,1,1,1]$. Then $\Phi^{\prime}$ is given by ${ }^{7}$ )

$\left.{ }^{7}\right)$ When there is no doubt about the game $v$ under consideration, we shall denote $\Phi^{\prime}(P, v)$ by $\Phi^{\prime}(P)$ which is consistent with the established notation. 


$$
\Phi^{\prime}(P)= \begin{cases}(3 / 6,1 / 6,1 / 6,1 / 6) & \text { if } P=(1234) \\ (3 / 5,1 / 5,1 / 5,0) & \text { if } P=(123)(4) \\ (3 / 4,1 / 4,0,0) & \text { if } P=(12)(3)(4) \text { or }(12)(34) \\ (0,1 / 3,1 / 3,1 / 3) & \text { if } P=(1)(234)\end{cases}
$$

For all other c.s.'s, $\Phi^{\prime}(P)$ can be determined by the symmetry of players 2,3 , and 4 . If is clear that $K_{0}\left(\Phi^{\prime}\right)=\{(1)(234)\}$. Note that in this example $t=2$, hence (1) $(234) \notin \Pi_{t}$.

Let

$$
s=\min _{R \in W^{m}} \sum_{i \in R} \phi_{i}(v)
$$

and let

$$
\Pi_{s}=\left\{P \in \Pi: P \text { contains a coalition } R \in W^{m} \text { such that } \sum_{i \in R} \phi_{i}(v)=s\right\} .
$$

Then we have the following important fact.

Theorem 7.8. Let $\Gamma$ be a proper monotonic simple game. Then $K_{0}\left(\Phi^{\prime}\right)=\Pi_{s}$.

Proof: Denote $\Phi^{\prime}((N))$ by $\phi=\left(\phi_{1}, \ldots, \phi_{n}\right)$. Let $P_{1} \in \Pi_{s}$. Suppose $P_{2} \in \Pi$ such that $P_{2} \operatorname{dom}_{R}\left(\Phi^{\prime}\right) P_{1}$ for some $R \in P_{2}$ such that $R \in W^{m}$. Then $\Phi^{\prime}\left(P_{2}\right)(i)>\Phi^{\prime}\left(P_{1}\right)(i)$ for all $i \in R$. Let $T \in P_{1}$ be such that $T \in W^{m}$ and $\sum_{i \in T} \phi_{i}=s$. Since $\Gamma$ is proper $R \cap T \neq \emptyset$. Pick $j \in R \cap T$. Then $\Phi^{\prime}\left(P_{1}\right)(j)=\phi_{j} / s$. Since $j \in R, \Phi^{\prime}\left(P_{2}\right)(j)=$ $=\phi_{j} /\left(\sum_{i \in R} \phi_{i}\right)>\phi_{j} / s$, i.e., $\sum_{i \in R} \phi_{i}<s$, a contradiction! Hence $K_{0}\left(\Phi^{\prime}\right) \supset \Pi_{s}$.

Let $P_{1} \in \Pi_{s}$ and $P_{2} \in \Pi$ be such that $P_{2} \notin \Pi_{s}$. Then $P_{1} \operatorname{dom}_{T}\left(\Phi^{\prime}\right) P_{2}$ where $T \in P_{1}$ such that $T \in W^{m}$ and $\sum_{i \in T} \phi_{i}=s$ because $\Phi\left(P_{1}\right)(i)=\phi_{i} / s$ for all $i \in T$ and $\Phi^{\prime}\left(P_{2}\right)(i)<\phi_{i} / s$ for all $i \in T$. Hence $K_{0}\left(\Phi^{\prime}\right) \subset \Pi_{s}$.

\section{Solutions with Respect to the Bargaining Set $M_{1}^{(i)}$}

The bargaining set was first introduced by Aumann/Maschler [1964]. They defined several types of bargaining sets. One of these, denoted by $M_{1}^{(i)}$, was shown to be nonempty for every c.s. by Peleg [1967].

Let $x^{R}$ denote a vector in $E^{r}$ where $r=|R|$, whose elements are indexed by the players in $R$. Let $x \in I(P)$ and let $i$ and $j$ be two distinct players in coalition $P_{k} \in P$. An objection of $i$ against $j$ to $x \in I(P)$ is a vector $y^{R}$, where $R$ is a coalition containing player $i$ but not $j$, whose coordinates $y_{l}$ satisfy $y_{i}>x_{i}, y_{l} \geqslant x_{l} \forall l \in R$ and $\sum_{l \in R} y_{l}=v(R)$. A counter-objection to this objection is a vector $z^{D}$, where $D$ is a coalition containing player $j$ but not $i$, whose coordinates $z_{l}$ satisfy $z_{l} \geqslant x_{l}$ for each $l \in D$, 
$z_{l} \geqslant y_{l}$ for each $l \in R \cap D$, and $\sum_{l \in D} z_{l}=v(D)$.

$x \in I(P)$ is stable if for each objection to $x$, there is a counter-objection. The bargaining set corresponding to the c.s. $P \in \Pi$, denoted by $M_{1}^{(i)}(P)$ is the set of all stable individually rational payoff $x \in I(P)$, i.e.,

$$
M_{1}^{(i)}(P)=\{x \in I(P): x \text { is stable }\}
$$

Theorem 8.1. Let $\Gamma$ be an $n$-person cooperative game with side payments. Then $M_{1}^{(i)}(P) \neq \emptyset$ for each $P \in \Pi$.

Proof. See Davis/Maschler [1967] and Peleg [1967].

As a result $\Pi\left(M_{1}^{(i)}\right)=\Pi$. The bargaining set is a natural payoff solution concept to study the solutions $J_{0}$ and $K_{0}$ for the following reasons:

(i) the bargaining set for each c.s. consists of payoffs that are stable in the sense of objections and counter-objections. If for a particular c.s., a payoff is not in the bargaining set, some player would have a justified objection (an objection that has no counter-objection) which when carried out would result in breakup of the coalition structure. Hence we are not justified in using unstable payoffs corresponding to a c.s. to dominate another c.s. Also,

(ii) the bargaining set is nonempty for each coalition structure.

We shall now determine $K_{0}\left(M_{1}^{(i)}\right)$ for all 3-person games with side payments.

Consider the 3-person game given by $N=\{1,2,3\}$,

$$
v(1)=v(2)=v(3)=0, v(12)=a, v(13)=b, v(23)=c,
$$

and

$$
v(123)=d, \text { where } 0 \leqslant a \leqslant b \leqslant c \text { and } d \geqslant 0 .
$$

Theorem 8.2. Let $\Gamma$ be a 3-person game as in (8.2) with $d>(a+b+c) / 2$.

(i) If $d<c$, then $K_{0}\left(M_{1}^{(i)}\right)=\{(1)(23)\}$

(ii) If $d=c$, then $K_{0}\left(M_{1}^{(i)}\right)=\{(1)(23),(123)\}$

(iii) If $d>c$, then $K_{0}\left(M_{i}^{(i)}\right)=\{(123)\}$.

\section{Proof:}

(i) In this case we have $(a+b) / 2+c / 2<d<c / 2+c / 2$, hence $a+b<c$. The bargaining set is given by

$$
M_{1}^{(i)}(P)= \begin{cases}(0,0,0) & \text { if } P=(1)(2)(3), \\ (0, a, 0) & \text { if } P=(12)(3), \\ (0,0, b) & \text { if } P=(13)(2), \\ \operatorname{Conv}\{(0, c-b, b),(0, a, c-a)\} & \text { if } P=(1)(23), \\ (0, d / 2-(b-a) / 2, d / 2+(b-a) / 2) & \text { if } P=(123)\end{cases}
$$


Clearly (1) (23) $\operatorname{dom}\left(M_{1}^{(i)}\right)(12)(3)$ and (1) (23) $\operatorname{dom}\left(M_{1}^{(i)}\right)(13)$ (2). Also since $(0, c / 2-(b-a) / 2, c / 2+(b-a) / 2) \in M_{1}^{(i)}((1)(23))$ and $c>d$,

(1) (23) $\operatorname{dom}\left(M_{1}^{(i)}\right)$ (123). The transition graph is shown in Figure 8.1. Hence Case (i) follows.

(ii) In this case, the bargaining set is as in (8.3) except for c.s. (123) which is

$M_{1}^{(i)}((123))=M_{1}^{(i)}((1)(23))$.

Therefore (ii) follows. (See Figure 8.2.)

(iii) Case 1) $c>a+b$

Here the bargaining set is as in (8.3) except for c.s. (123) which is given by

$M_{1}^{(i)}((123))=\left\{\left(x_{1}, x_{2}, x_{3}\right): x_{1}+x_{2} \geqslant a, x_{1}+x_{3} \geqslant b, x_{2}+x_{3} \geqslant c\right.$, and

$x_{1}+x_{2}+x_{3}=d$.

For each $\left(0, x_{2}, c-x_{2}\right) \in M_{1}^{(i)}((1)(23))$ where $a \leqslant x_{2} \leqslant c-b$, we have $\left((d-c) / 3, x_{2}+(d-c) / 3, c-x_{2}+(d-c) / 3\right) \in M_{1}^{(i)}((123))$. Hence (123) dom $\left(M_{1}^{(i)}\right)(1)(23)$. The transition digraph is shown in Figure 8.3.

(iii) Case 2) $c \leqslant a+b$

In this case the bargaining set is given by

$$
M_{1}^{(i)}(P)= \begin{cases}(0,0,0) & \text { if } P=(1)(2)(3), \\ \left(p_{1}, p_{2}, 0\right) & \text { if } P=(12)(3), \\ \left(p_{1}, 0, p_{3}\right) & \text { if } P=(13)(2), \\ \left(0, p_{3}, p_{3}\right) & \text { if } P=(1)(23), \\ \operatorname{Co}((123)) & \text { if } P=(123) .\end{cases}
$$

where $p_{1}=(a+b-c) / 2, p_{2}=(a+c-b) / 2, p_{3}=(b+c-a) / 2$, and

$\operatorname{Co}((123))=\left\{\left(x_{1}, x_{2}, x_{3}\right): x_{1}+x_{2} \geqslant a, x_{1}+x_{3} \geqslant b, x_{2}+x_{3} \geqslant c\right.$, and $\left.x_{1}+x_{2}+x_{3}=d\right\}$.

Let $p=\left(p_{1}+p_{2}+p_{3}\right)$, then clearly, $\left(p_{1}+(d-p) / 3, p_{2}+(d-p) / 3, p_{3}+(d-p) / 3\right) \in M_{1}^{(i)}((123))$

Hence c.s. (123) dominates (w.r.t. $M_{1}^{(i)}$ ) every other c.s. This case completes the proof of the theorem.

Theorem 8.3. Let $\Gamma$ be a 3-person game as in (8.2) with $d=(a+b+c) / 2$.

(i) If $c \leqslant a+b$ then $K_{0}\left(M^{(i)}\right)=\{(12)$ (3), (13) (2), (1) (23), (123) $\}$.

(ii) If $c>a+b$ then $K_{0}\left(M_{i}^{(i)}\right)=\{(1)(23)\}$.

\section{Proof:}

(i) In this case, the bargaining set is as in (8.4) with $M_{1}^{(i)}((123))=\left(p_{1}, p_{2}, p_{3}\right)$. The result clearly follows.

(ii) In this case, the bargaining set is as in (8.3). Since $d<c$, the result follows. 


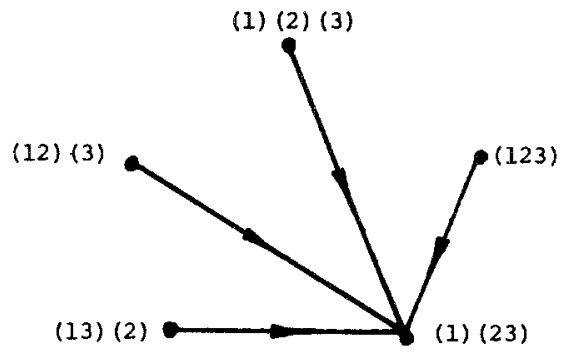

Fig. 8.1: The transition digraph in Theorem 8.2, (i), and in Theorem 8.4, (ii)

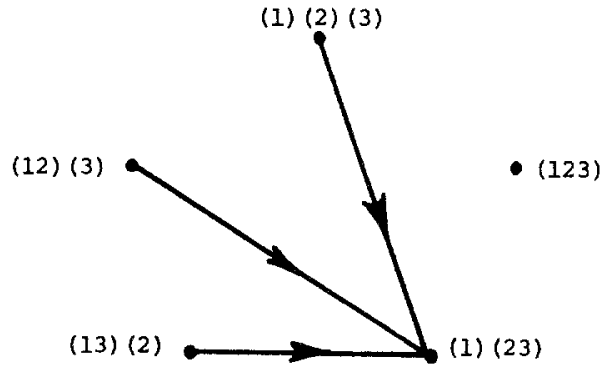

Fig. 8.2: The transition digraph in Theorem 8.2, (ii)

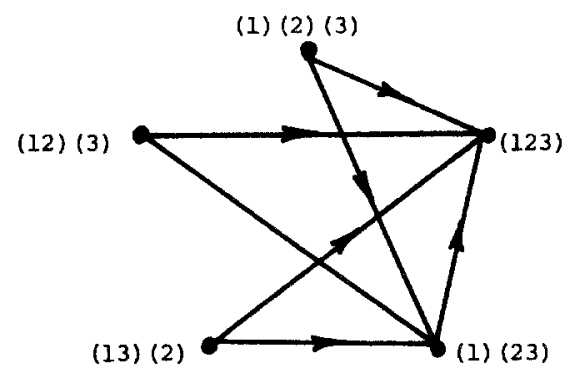

Fig. 8.3: The transition digraph in Theorem 8.2, (iii) case 1)

Theorem 8.4. Let $\Gamma$ be a 3-person game as in (8.2), with $d<(a+b+c) / 2$.

(i) If $c \leqslant a+b$ then $K_{0}\left(M_{1}^{(i)}\right)=\{(12)(3),(13)(2),(1)(23)\}$.

(ii) If $c>a+b$ then $K_{u}\left(M_{1}^{(i)}\right)=\{(1)(23)\}$.

Proof:

(i) In this case, the bargaining set is as in (8.4) except for c.s. (123) for which it is given by 


$$
M_{1}^{(i)}((123))=\left\{\begin{array}{cc}
\left(p_{1}+(d-p) / 3, p_{2}+(d-p) / 3,\right. & \left.p_{3}+(d-p) / 3\right) \\
& \text { if } 2 c-a-b \leqslant d \\
(0, d / 2-(b-a) / 2, d / 2+(b-a) / 2) & \text { if } b-a \leqslant d<2 c-a-b \\
(0,0, d) & \text { if } d<b-a .
\end{array}\right.
$$

In all cases, the transition graph is presented in Figure 8.4. Therefore (i) follows.

(ii) In this case the bargaining set is as in (8.3) except for c.s. (123) for which the bargaining set is as in (8.5). The transition graph is shown in Figure 8.1. Hence the result follows.

(12) (3)

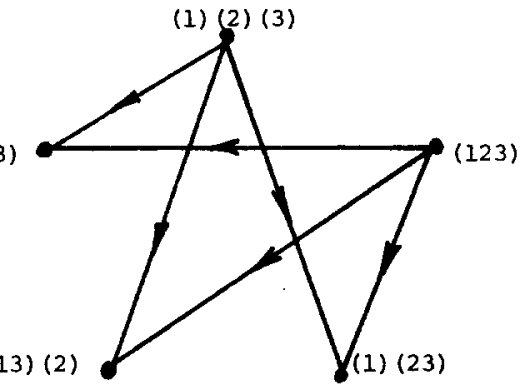

Fig. 8.4: The transition graph in Theorem 8.4, (i)

Since Theorems $8.2,8.3$ and 8.4 cover all cases, we have proved the following.

Theorem 8.5. Let $\Gamma$ be a 3-person game as in (8.2). Then $K_{0}\left(M_{1}^{(i)}\right) \neq \emptyset$.

For every $P \in \Pi$, if $x \in I(P)$ belongs to $\operatorname{Co}(P)$, then no player can have an objection against another player. Thus if $\operatorname{Co}(P) \neq \emptyset, \operatorname{Co}(P) \subset M_{1}^{(i)}(P)$. Hence the p.s.c. $M_{1}^{(i)}$ satisfies the hypothesis of Corollary 6.2. So we obtain the following.

Lemma 8.6. Let $\Gamma$ be an $n$-person game. If $\Pi(\mathrm{Co}) \neq \emptyset$ then $K_{0}\left(M_{1}^{(i)}\right) \neq \emptyset$. In fact $K_{0}\left(M_{1}^{(i)}\right) \supset \Pi_{z}$.

Proof: This is a consequence of Corollary 6.2 and Theorem 6.3.

No general existence theorem for $K_{0}\left(M_{1}^{(i)}\right.$ is known at this time. Example 8.1 illustrates a pathology for $K_{0}\left(M_{1}^{(i)}\right)$ which is due to a "flaw" in the properties of the bargaining set.

Example 8.1. Let $\Gamma$ be a 3-person veto player game with

$$
v(12)=v(13)=v(123)=1, v(R)=0 \text { for all other } R \subset N .
$$

Observe that player 1 is a veto player because all coalitions that doe not contain player 1 are losing coalitions. However, player 1 is not a dictator since he needs either player 2 or player 3 (or both) to form a winning coalition. The bargaining set of this game is given by 


$$
M_{1}^{(i)}(P)= \begin{cases}(1,0,0) & \text { if } P=(123),(12)(3) \text { or }(13)(2) \\ (0,0,0) & \text { for all other } P \text { in } \Pi .\end{cases}
$$

Note that in every c.s. that contains a coalition which has a positive value, at least one player in the coalition gets zero payoff in the bargaining set. As a result, due to Condition (3.5) in the definition of domination, no c.s. dominates another c.s. Hence $K_{0}\left(M_{1}^{(i)}\right)=\Pi$.

The above example exhibits a flaw in the properties of the bargaining set. E.g., in the c.s. (12) (3) player 2 gets zero payoff in the bargaining set. This is because player 2 has no 'bargaining power' at all vis-á-vis player 1 . Since there are no coalitions with a positive value that contains player 2 but not player 1 , player 2 cannot even object! However the payoff in the bargaining is counter-intuitive because we could argue: Why should player 2 enter into a coalition with player 1 if his share of the resulting coalitional value is the same as what the player could have obtained had he been in a coalition by himself? In this respect, we could say that the bargaining set is derived entirely from the bargaining positions of the players in the process of coalition formation in contrast with the Shapley value which is derived entirely from the characteristic function of the game. These two p.s.c.'s reflect two extreme view points in looking at solutions of cooperative games in characteristic function form. A major research problem is to define a p.s.c. that exhibits both the strategic value and the bargaining power of the players.

One method of attacking this problem in the case of the bargaining set is to regard the bargaining set as an idealization (of the bargaining process) and relax the definition of an objection by $\epsilon$, where $\epsilon$ is a small positive real number. More formally, let $x \in I(P)$ and $i$ and $j$ be two distinct players in a coalition $P_{k} \in P$. An $\epsilon$-objection of $i$ against $j$ is a vector $y^{R}$, where $R$ is a coalition containing player $i$ but not $j$, whose coordinates $y_{l}$ satisfy $y_{i}>x_{i}+\epsilon, y_{l} \geqslant x_{l}$ for all $l \in R$, and $\sum_{l \in R} y_{l}=v(R)$. A counterobjection to this $\epsilon$-objection is defined as before. We say $x \in I(P)$ is $\epsilon$-stable if for each $\epsilon$-objection in $x$, there is a counter-objection. The $\epsilon$-bargaining set, denoted by $M_{1, \epsilon}^{(i)}$, corresponding to c.s. $P \in \Pi$ is the set of all $\epsilon$-stable $x \in I(P)$, i.e.,

$$
M_{1, \epsilon}^{(i)}(P)=\{x \in I(P): x \text { is } \epsilon \text {-stable }\} .
$$

We could regard $\epsilon$ as a 'sacrifice' each player is willing to make (if necessary) for coalitional stability.

Note that the results in Theorems 8.2, 8.3, 8.4 and 8.5 as well as Lemma 8.6 remain unchanged if we replace $M_{1}^{(i)}$ by $M_{1, \epsilon}^{(i)}$.

Example 8.2. Consider the game in Example 8.1. The $\epsilon$-bargaining set is given by

\footnotetext{
${ }^{8}$ ) Denotes the set $\left\{\left(x_{1}, x_{2}, x_{3}\right): x_{1}=1-x_{2}-x_{3}, 0 \leqslant x_{2} \leqslant \epsilon, 0 \leqslant x_{3} \leqslant \epsilon\right\}$.
} 


$$
M_{1, \epsilon}^{(i)}(P)= \begin{cases}\left(1-x_{2}-x_{3}, 0 \leqslant x_{2} \leqslant \epsilon, 0 \leqslant x_{3} \leqslant \epsilon\right) & \text { if } \left.^{8}\right) P=(123) \\ \left(1-x_{2}, 0 \leqslant x_{2} \leqslant \epsilon, 0\right) & \text { if } P=(12)(3) \\ \left(1-x_{3}, 0,0 \leqslant x_{3} \leqslant \epsilon\right) & \text { if } P=(13)(2) \\ (0,0,0) & \text { for all other } P \text { in } \Pi .\end{cases}
$$

It is clear that $K_{0}\left(M_{1, \epsilon}^{(i)}\right)=\{(123),(12)(3),(13)(2)\}$ which is more intuitive than $K_{0}\left(M_{1}^{(i)}\right)=\Pi$.

Example 8.3. (The Chemical Company Game. See Anderson/Traynor [1962]). Two chemical companies $C_{1}$ and $C_{2}$ supply two fabricating companies $F_{1}$ and $F_{2}$. The permissible coalition structures are:

$$
\begin{array}{ll}
P_{1}=\left(C_{1}\right)\left(C_{2}\right)\left(F_{1}\right)\left(F_{2}\right), & P_{2}=\left(C_{1} F_{1}\right)\left(C_{2}\right)\left(F_{2}\right), \\
P_{3}=\left(C_{1} F_{2}\right)\left(C_{2}\right)\left(F_{1}\right), & P_{4}=\left(C_{1}\right)\left(C_{2} F_{1}\right)\left(F_{2}\right), \\
P_{5}=\left(C_{1}\right)\left(C_{2} F_{2}\right)\left(F_{1}\right), & P_{6}=\left(C_{1} F_{1}\right)\left(C_{2} F_{2}\right), \\
P_{7}=\left(C_{1} F_{2}\right)\left(C_{2} F_{1}\right) . &
\end{array}
$$

The respective payoffs (profits) to these coalitions in the particular coalition structures are:

$$
\begin{array}{ll}
P_{1}: 25,15,75,100 . & P_{2}: 300,25,110 . \\
P_{3}: 500,30,85 . & P_{4}: 28,200,105 . \\
P_{5}: 30,425,90 . & P_{6}: 400,600 . \\
P_{7}: 700,300 . & \cdots
\end{array}
$$

This "partition function" induces the characteristic function:

$$
\begin{aligned}
& v\left(C_{1}\right)=25, v\left(C_{2}\right)=15, v\left(F_{1}\right)=75, v\left(F_{2}\right)=100, v\left(C_{1}, F_{1}\right)=300, \\
& v\left(C_{1}, F_{2}\right)=500, v\left(C_{2}, F_{1}\right)=200, v\left(C_{2}, F_{2}\right)=425 .
\end{aligned}
$$

The bargaining set $M_{1}^{(i)}$ is given by

$$
M_{1}^{(i)}(P)= \begin{cases}(25,15,75,100) & \text { if } P=P_{1} \\ \left(115 \leqslant x_{1} \leqslant 225,15,300-x_{1}, 100\right) & \text { if } P=P_{2} \\ \left(90 \leqslant x_{1} \leqslant 225,15,75,500-x_{1}\right) & \text { if } P=P_{3} \\ \left(25,15 \leqslant x_{2} \leqslant 125,200-x_{2}, 100\right) & \text { if } P=P_{4} \\ \left(25,15 \leqslant x_{2} \leqslant 125,75,425-x_{2}\right) & \text { if } P=P_{5} \\ \left(x_{1}, x_{2}, 300-x_{1}, 425-x_{2}\right) & \text { if } P=P_{6} \\ \text { where } x_{1}, x_{2} \text { are as in Figure 8.5. } & \\ \left(y_{1}, y_{2}, 200-y_{2}, 500-y_{1}\right) & \text { if } P=P_{7} \\ \text { where } y_{1}, y_{2} \text { are as in Figure 8.6. } & \end{cases}
$$



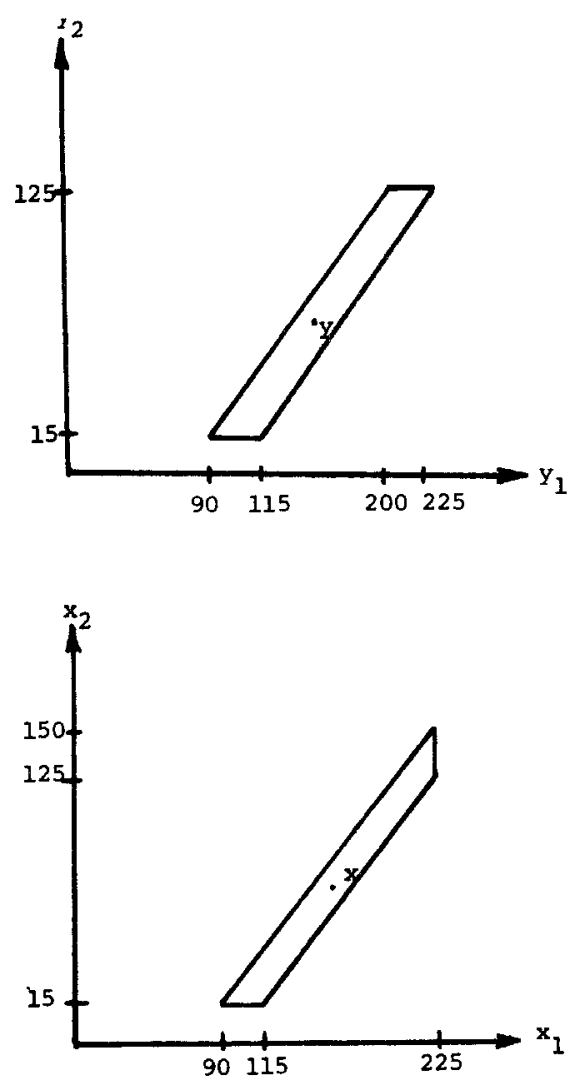

Fig. 8.5: The bargaining set $M_{1}^{(i)}\left(P_{6}\right)$ for the chemical company game
Fig. 8.6: The bargaining set $M_{1}^{(i)}\left(P_{7}\right)$ for the chemical company game

The transition digraph is shown in Figure 8.7. Hence $K_{0}\left(M_{1}^{(i)}\right)=\left\{\left(C_{1} F_{1}\right)\left(C_{2} F_{2}\right)\right.$, $\left.\left(C_{1} F_{2}\right)\left(C_{2} F_{1}\right)\right\}$.

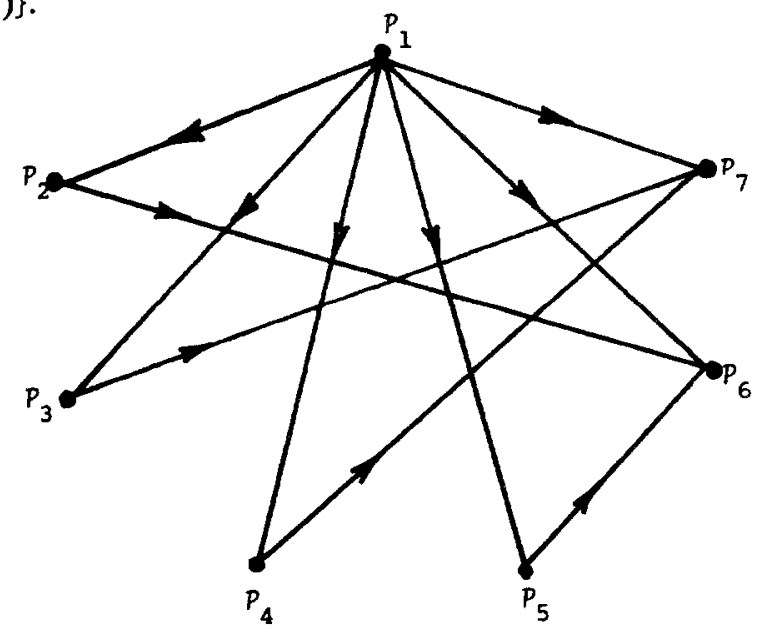

Fig. 8.7: The transition digraph of the chemical company game 


\section{Some Modifications of the Coalition Structure Model}

In this section, we look at some modifications of the domination relation in the abstract game $(\Pi(S), \operatorname{dom}(S))$. We define two other domination relations one of which is stronger than $\operatorname{dom}(S)$ and the other weaker than $\operatorname{dom}(S)$.

Definition 9.1. Let $P_{1}, P_{2} \in \Pi(S)$ and $S$ be a p.s.c. Then $P_{1}$ weakly dominates $P_{2}$, denoted by $P_{1} w$-dom $(S) P_{2}$, iff

$$
\begin{aligned}
& \text { for each } y \in S\left(P_{2}\right), \exists \text { a nonempty } R \in P_{1} \text { and } x \in S\left(P_{1}\right) \\
& \text { such that } x_{i}>y_{i} \text { for all } i \in R \text {. }
\end{aligned}
$$

Definition 9.2. Let $P_{1}, P_{2} \in \Pi(S)$ and $S$ be a p.s.c. Then $P_{1}$ strongly dominates $P_{2}$, denoted by $P_{1} s$-dom $(S) P_{2}$, iff $\exists$ a nonempty $R \in P_{1}$ and $x \in S\left(P_{1}\right)$ such that for all $y \in S\left(P_{1}\right), x_{i}>y_{i}$ for all $i \in R$.

The following relations are direct consequence of Definitions 3.6, 9.1 and 9.2.

$$
\begin{aligned}
& \text { If } P_{1} s-\operatorname{dom}(S) P_{2} \text {, then } P_{1} \operatorname{dom}(S) P_{2} \text {. } \\
& \text { If } P_{1} \operatorname{dom}(S) P_{2} \text {, then } P_{1} w-\operatorname{dom}(S) P_{2}
\end{aligned}
$$

Let $K_{0, w}(S)$ and $K_{0, s}(S)$ denote the cores of the abstract games (I(S),w-dom(S)) and $(\Pi(S)$, s-dom(S)) respectively. As a consequence of Relations (9.2) and (9.3), we have

$$
K_{0, s}(S) \supset K_{0}(S) \supset K_{0, w}(S) \text {. }
$$

Also, if $S$ is a p.s.c. such that for each $P \in \Pi, S(P)$ is either a single point set in $E^{n}$ or an empty set, then

$$
K_{0, s}(S)=K_{0}(S)=K_{0, w}(S)
$$

\section{Appendix: The Aumann-Dreze Generalization of the Shapley Value for all Simple Games with Four or Fewer Players}

The table on the following page contains all distinct proper simple games of four or fewer players excluding dummies. All winning coalitions are listed - the minimal winning coalitions are listed first and separated from the rest by a semicolon. The weighted voting representation given in column 4 are the simplest ones. The Shapley value $\Phi$ of a c.s. depends only on the winning coalition contained in the c.s. The Shapley value of all c.s.'s containing winning coalitions, in the sequence as in column 3 , is given in column 5. The Shapley value of a c.s. not containing any winning coalition is zero for each player and therefore is not given in column 5 . Column 6 contains all c.s.'s in $K_{0}(\Phi)$ identified by the winning coalition it contains. The last column indicates whether the game exhibits the paradox of smaller coalitions or not. 


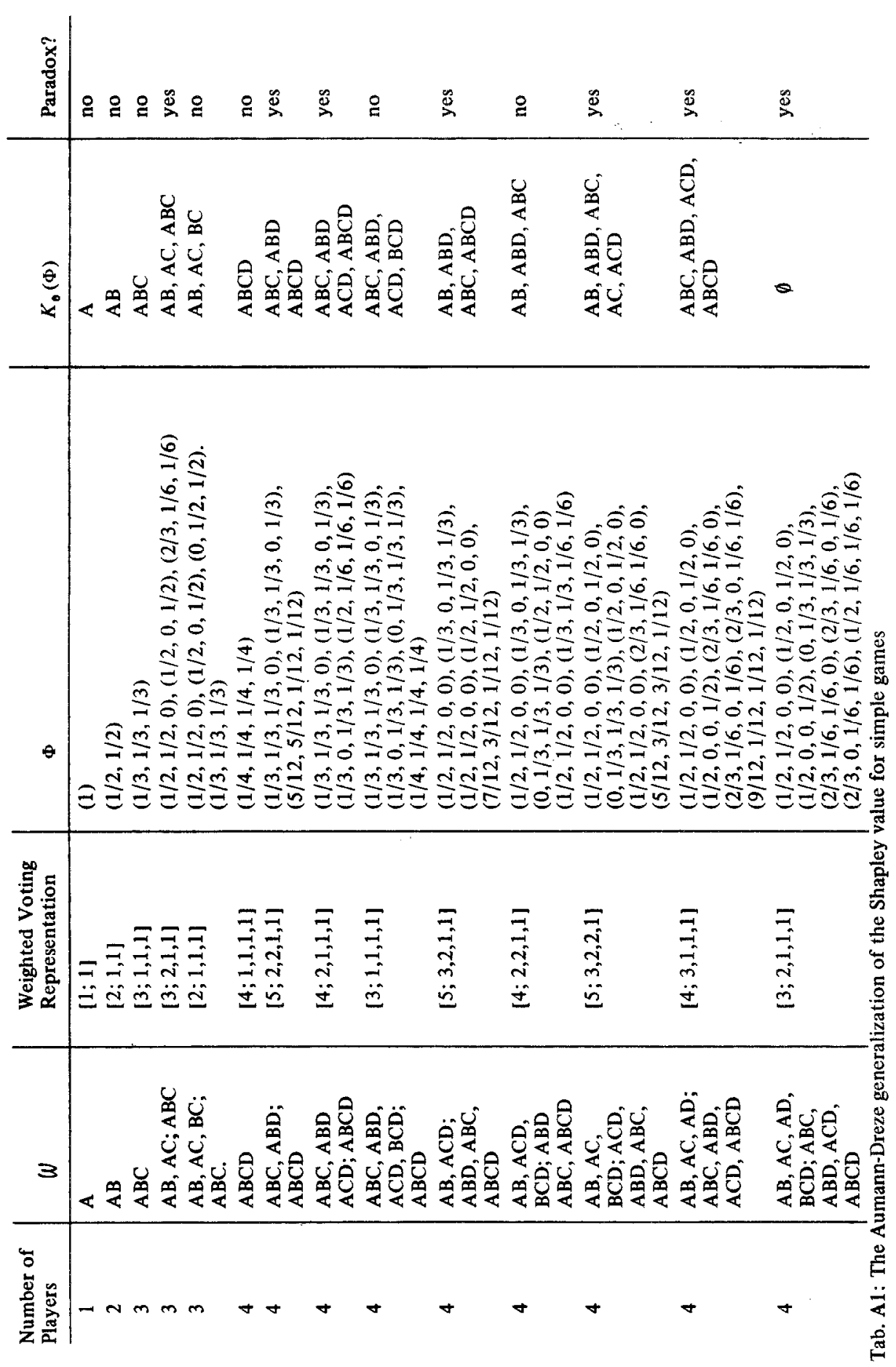




\section{References}

Anderson, S.L., and E.A. Traynor: An application of the Aumann-Maschler $n$-person cooperative game. Recent Advances in Game Theory. Ed. by M. Maschler. Princeton 1962, 265-270.

Aumann, R.J., and $J$. Dreze: Cooperative games with coalition structures. International Journal of Game Theory 3, 1974, 217-237.

Aumann, R.J., and M. Maschler: The bargaining set for cooperative games. Annals of Mathematics Study 52, 1964, 443-476.

Bondareva, O.N.: Theory of the core in the $n$-person game. Leningrad State University Vestnik L. G. U. 13, 1962, 141-142. (In Russian.)

-: Some applications of linear programming methods to the theory of cooperative games. Problemy Kibernetiki 10, 1963, 119-139. (In Russian.)

Brams, S.J.: Paradoxes in Politics. New York 1976.

Davis, $M$., and $M$. Maschler: Existence of stable payoff configurations fot cooperative games. Essays in Mathematical Economics in Honor of Oskar Morgenstern. Ed. by M. Shubik. Princeton 1967, 39-52.

Gamson, W.A.: A theory of coalition formation. American Sociological Review 26, 1961, 373-382.

Gillies, D.B.: Solutions to general non-zero-sum games. Annals of Mathematics Study 40, 1959, $47-85$.

Harary, F.: Graph Theory. Massachusetts 1959.

Kalai, E., E.A. Pazner and D. Schmeidler: Collective choice correspondences as admissible outcomes of social bargaining processes. Econometrica 44, 1976, 223-240.

Lucas, W.F.: A game with no solution. Bull. Amer. Math. Soc. 74, 1968, 237-239.

-: A proof that a game may not have a solution. Trans Amer. Math. Soc. 137, 1969, 219-229.

Lucas, W.F., and J.C. Maceli: Discrete partition function games. Game Theory and Political Science. Ed. by P. Ordeshook. New York 1978, 191-213.

Peleg, B.: Existence theorem for the bargaining set. Essays in Mathematical Economics in Honor of Oskar Morgenstern. Ed. by M. Shubik. Princeton 1967, 53-76.

Riker, W.H., and P. Ordeshook: An Introduction to Positive Political Theory. New York 1973.

Shapley, $L . S$. : A value for $n$-person games. Annals of Mathematics Study 28, 1953, 307-317.

-: Simple games: An outline of the descriptive theory. Behavioral Science 7, 1962, 59-66.

-: On balanced sets and cores. Naval Research Logistics Quarterly 14, 1967, 453-460.

Shapley, L.S., and M. Shubik: A method for evaluating the distribution of power in a committee system. Amer. Political Science Review 48, 1954, 787-792.

Shenoy, P.P.: A dynamic solution concept for abstract games. Mathematics Research Center, Technical Summary Report No. 1804, University of Wisconsin, Madison 1977a.

-: On game theory and coalition formation. School of Operations Research and Industrial Engineering, Cornell University, Technical Report No. 342, New York, $1977 \mathrm{~b}$.

--: On coalition formation in simple games: A mathematical analysis of Caplow's and Gamson's theories. Journal of Mathematical Psychology 18, 1978, 177-194.

Thrall, R.M., and W.F. Lucas: $n$-person games in partition function form. Naval Research Logistics Quarterly 10, 1963, 281-298.

Von Neumann, J., and O. Morgenstern: Theory of Games and Economic Behavior. Princeton 1944, 3rd edition, 1953.

Received May, 1978

(revised version June, 1979) 